\title{
12. DETERMINING PRODUCT PLATFORM EXTENT
}

\author{
Olivier L. de Weck ${ }^{*}$
}

\section{INTRODUCTION}

Typically, it is assumed that a product family will be derived from a single platform once a firm has decided on platforming as an appropriate strategy. The totality of all variants represented in a single-platform family defines the lower and upper performance and value bounds which have to be supported by the platform. This difference between upper and lower bounds of a platform is commonly referred to as platform extent (Seepersad, Hernandez, Allen, 2000). However, the average number of variants built from a single platform has been steadily increasing in a number of industries (automotive, electronics, aircraft) since the early 1990's. Fig. 1 shows that the number of models per platform in the automotive industry has been increasing since 2002. This trend had started in the mid 1990's and is likely to continue in the future. The consequence is that each platform has to accommodate a larger number of variants, whereby the extent of the platform is constantly being challenged with each new variant that is assigned to it. There is general agreement that mass customization has led to an increasing fragmentation of the automotive market with the number of individual models for sale in the U.S. rising each year from 33 in 1947, to 198 in 1990 to an estimated 277 in 2009 (Simmons 2005).

\section{Average Vehicle Models per Platform}

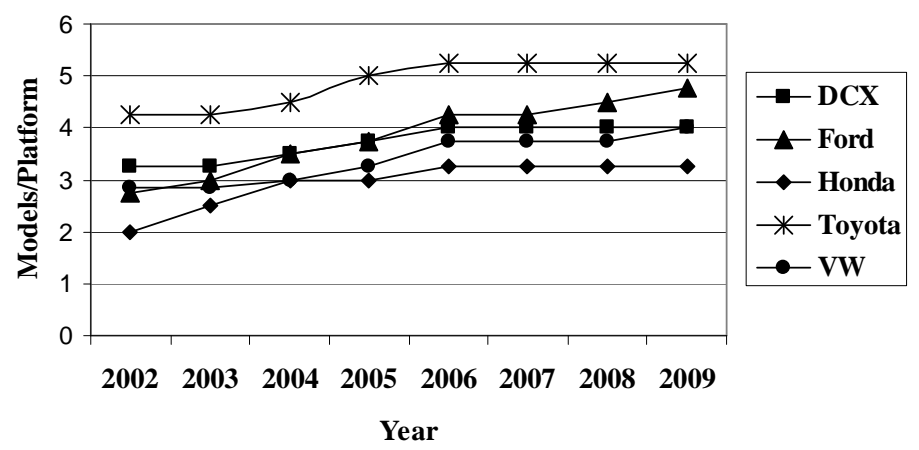

Figure 1. Variants per platform trend in the automotive industry (Source: PWC, 2003)

This pervasive phenomenon can be attributed to accelerating mass customization and concomitant market fragmentation. Recently, manufacturers have begun to realize that platforms cannot be "stretched" indefinitely, before the competitiveness of some of the associated variants is compromised in a competitive environment. This dilemma has

\footnotetext{
* Assistant Professor, Room 33-410, Dept. of Aeronautics \& Astronautics, Engineering Systems Division, Massachusetts Institute of Technology, Cambridge, MA 02139, U.S.A., telephone: (617) 253-0255, email: deweck@mit.edu
} 
given rise to research in the area of multi-platform strategy. The questions posed in this context are not too different from those surfacing in the classic tradeoff between component commonality and product distinctiveness when designing a single product platform. We want to know:

1. Given a set of $m$ product variants, what is the optimal number of platforms, $p$, to derive these from?

2. What is the optimal assignment of the $m$ product variants to the $p$ platforms, given a set of target market segments and competitors?

3. What criteria should be used to decide on platform extent?

The multi-platform problem manifests itself at the highest level in the system hierarchy going from individual components to modules and platforms to products to families-of-products. Deciding which components to make common and potentially include in a platform, and which ones to keep unique for each variant is primarily a problem addressed by product designers and manufacturing engineers within the firm (see other chapters). The platform extent problem, on the other hand, is of a more strategic nature since the question cannot be answered without at least a rudimentary understanding of the interplay between product architecture, manufacturing, cost, engineering performance, value, demand and the role of competition. Despite this domain-crossing complexity, we believe that the platform extent problem can be rigorously addressed both quantitatively and qualitatively. This is primarily so because the number of factors to consider are numerous, but finite. The primary challenge in solving the platform extent problem arises from questions of sufficient model fidelity and robustness (depth), rather than questions of comprehensiveness and completeness (breadth).

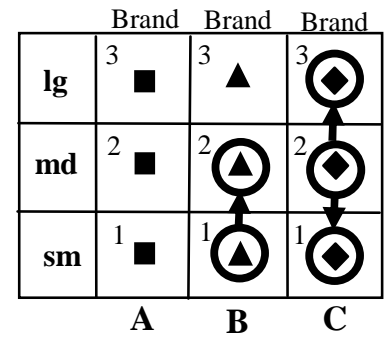

(a)

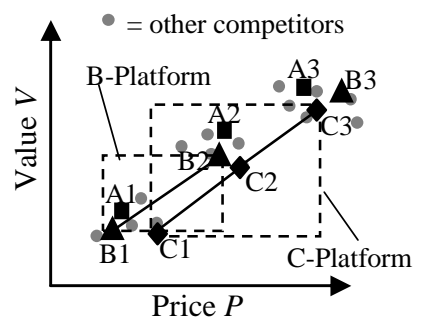

(b)

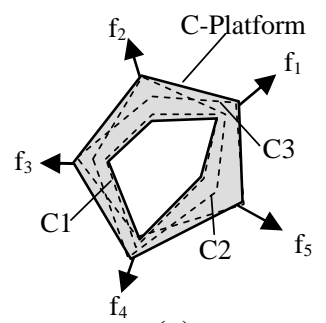

(c)

Figure 2. Visualization of the platform extent problem: (a) market segmentation grid with connected circles indicating a platform, (b) value-price positioning, (c) notional functional radar plot for C-platform showing platform extent

Fig. 2 visualizes the platform extent problem in three domains. First, one may investigate the assignment of variants to platforms in the context of platform leveraging strategies across a market segmentation grid (Fig. 2a). Here, we distinguish between three hypothetical manufacturers ("brands"), whereby A offers three variants (small, medium large) without platforming, B combines the first two products in a platform, but not the third, and firm $\mathrm{C}$ derives all three products from a common platform. Which of these three strategies is superior cannot be decided from the market segmentation grid alone. 
Plotting each product in the value-price diagram (Cook 1997) reveals where each product variant falls with respect to market segment averages and competitors. Platform extent can also be understood as the value-price envelope of all variants associated with a platform (Fig. 2b). In this case the C-platform covers the largest valueprice region (dashed box). Finally, Fig. $2 \mathrm{c}$ shows the functional performance envelope that contains all variants for the C-platform in a spider chart (radar plot). The gray shaded area can be interpreted as the functional extent of the platform. One can also think of platform extent in the design space (not shown), where the lower and upper bounds of key design variables of the product determine the limits of the platform ${ }^{\dagger}$.

This chapter presents a brief review of prior work on the multi-platform (extent) problem and discusses the relevance of the issue to industry (Section 2). Next, in Section 3 , we present a modeling framework for single products, which connects the following six domains: (1) product architecture, (2) engineering performance, (3) product value, (4) market demand, (5) manufacturing cost and (6) investment finance. We will argue that such a comprehensive modeling framework is not only desireable, but a necessary - if not sufficient - condition to address the platform extent question. Section 4 shows the extension of the single product model to the multiple-variant, but still single platform case. We will argue that the effect of platforming must be assessed both in terms of its external (market) as well as its internal (manufacturing) consequences. Once the number of variants increases, say beyond $m>3$, the question of multiple platforms must be addressed (Section 5). We develop the ideas in this chapter based on an actual database of vehicles sold in the U.S. market (2002). Conclusions are drawn in Section 6, whereby the robustness of the answers to various assumptions is discussed. Finally, we conclude with a number of reflections regarding the platform extent problem and recommended areas of future research.

\section{LITERATURE AND REFLECTIONS ON MULTIPLE PLATFORMS}

We attribute the first paper on the question of platform extent (multiple platforms) to Seepersad, Hernandez and Allen (2000). In their work they wrestled with the question of finding the optimal platform extent, and therefore the right number of platforms, for a family of industrial absorption chillers. Their method to determine platform extent uses a compromise Decision Support Problem, enhanced by linear physical programming. In this work a manufacturer seeks to offer a family of eight chillers in the capacity range of $600-1,300$ tons, in 100 ton increments, based on one or more platforms. Targets are set for manufacturing costs across the family, as well as cycle time for manufacturing of individual variants. A linear physical program attempts to minimize the compound deviation from these targets, $Z$. The quantity $Z$ represents a weighted sum across the nine targets and four desirability ranges of physical programming. The authors found that a single platform was satisfactory in cases of uniform demand distribution, but that two platforms were advantageous when significant demand gaps created a distance between

\footnotetext{
${ }^{\dagger}$ One has to be careful to distinguish between the actual platform extent, which is given by variants actually derived from a platform and the potential platform extent, which is defined by the set of variants that could potentially be derived, while still remaining competitive in the market place. The second kind is generally not known a priori.
} 
low and high capacity product variants. Their model addressed the domains of product architecture, engineering performance, manufacturing cost, and capital investment, but did not take into account the effects of customer valuation, market demand and competition.

This last point is important, since it is problematic to assess the potential benefits of a platform strategy in non-monopolistic markets without modeling competition as well. Cook (1997) reports that "Ironically, GM's market share relative to Ford only began to recede in the mid 1980s as GM's brands - Chevrolet, Pontiac, Oldsmobile, Buick, and Cadillac - became less distinctive through the use of common platforms and exterior stampings that reduced product differentiation."

We also found that much of the literature on the multi-platform problem is either engineering-centric or management-centric. It is apparent that engineers rarely have access to and expertise in market related topics. Conversely, management does not have a detailed understanding of the technical issues resulting from various platforming decisions. A practical as well as academic treatment of the platform extent problem needs to eventually bridge the gap between management and engineering.

The ultimate goal of product platforming is long-term profit maximization for the firm, leading to a sustainable increase in shareholder value. We argue that there can only be this one objective, when trying to address the single or multi-platform question. The next section presents a framework that models profit arising from a single product, without platforming. Subsequent sections extend this framework to multiple product variants and platforms.

\section{SINGLE PRODUCT MODEL}

\subsection{Reasoning and Modeling Framework}

Figure 3 shows a reasoning and modeling framework that helps clarify the primary domains that exist in the for-profit manufacturing firm. This view is product-centric and does not attempt to capture the myriad of organizational issues present in manufacturing firms. The figure shows the six primary domain blocks, as well as the main variables flowing between blocks in addition to important exogenous inputs. Exogenous inputs are those that are not under direct control of the firm.

First, product architecture (1) defines the value-generating functions of the product and maps these to physical components (parts) and modules which are assemblies of parts ${ }^{\ddagger}$. Inputs to product architecture are regulations and standards with which the machine must comply. The choice of operating principles of the machine and its decomposition relate the physical components to the vector of independent design variables, $\mathbf{x}$, for which engineers will find the most appropriate values. In order to accomplish this, engineering (2) creates models of functional product performance attributes, $\mathbf{f}$, as a function of the design variables, $\mathbf{x}$.

\footnotetext{
$\$$ Parts are the atomic units of the product, which cannot be further taken apart before
} they loose their functionality and integrity. Parts can be hardware or software. 


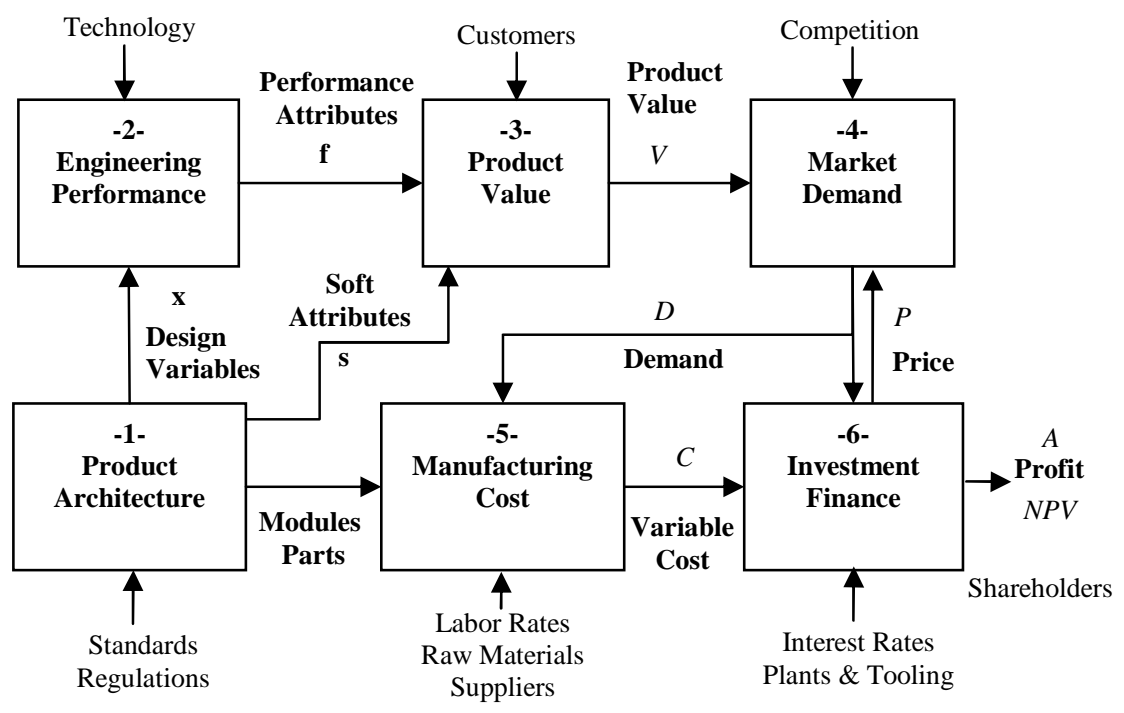

Figure 3. Single product reasoning and modeling framework

The interface between engineering and marketing is primarily concerned with how the vector of performance attributes, $\mathbf{f}$, translates to value, $V$, in the marketplace. The product value model (3) is also impacted by soft attributes, s, such as styling, comfort, or dependability, which are only measurable via customer surveys but not directly via (physics-based) performance attributes and engineering models. We subscribe to Cook's (1997) view that value is to be measured in the same monetary unit as price, e.g. [\$]. Given a database of existing products grouped into market segments, and given transaction prices, $P$, demand, $D$, functional attributes, $\mathbf{f}$, and soft attributes, $\mathbf{s}$, for these products one can estimate both the predicted value of each product in the marketplace, as well as the relative contribution that each of the functional and soft attributes make to that value. Demand and market share estimation (4) in non-monopolistic markets requires the same information about the competitor's products as about ones own products. This is often not fully available, but specialized service providers (e.g. J.D. Power), government agencies and corporate intelligence are usually in a position to provide ample data in the aforementioned categories. This is particularly true for well established markets (e.g. automobiles, household appliances) but less true for emerging markets (e.g. digital portable devices). It has been our experience that paucity of data is not usually the main problem in enterprises that consider establishing or refining a successful product family strategy. Rather, what is missing is a coherent end-to-end framework, such as the one shown in Figure 3, to integrate and interpret the available data. Of all the disciplines involved, demand estimation is the most difficult and uncertain, but it is essential. Much of the engineering literature on platforms bypasses this difficulty by resorting to constructs such as "acceptable performance losses due to platforming", thereby ignoring true market effects. While such simplifying assumptions are understandable in the context of academic research, the for-profit manufacturing firm does not have the luxury of drawing such arbitrary boundaries between domains. 
It is interesting to note that when actual sales volumes are available for market segments of interest (actual demand $D_{a}$ ), one has an opportunity to infer the relative weighting factors, $\gamma$, between the functional and soft attributes by minimizing the difference between predicted and actual demand. This will be shown later.

Once demand, $D$, has been estimated in this fashion one can derive the production volume, $D_{p}$, which is simply the predicted demand plus scrap and surplus. While discussing platform extent in this chapter, we will assume that demand and production volume are identical. Given labor and material rates, one follows the lower branch in Fig. 3 to estimate the variable (per unit) manufacturing cost, $C$, for the products of interest (5). This estimate relies on knowledge of the product architecture, i.e. sets of modules and parts that make up the product as well as a determination of the main fabrication and assembly steps. Estimation of the profit, $A$, also requires an estimate of the fixed manufacturing cost, $F$, which - to first order - are assumed to be independent of production quantity, demand $D$, as well as the investment cost, $M$. This is done in the investment finance block (6), thus completing the modeling framework. Having obtained estimates for demand, $D$, price, $P$, variable cost, $C$, fixed cost, $F$, and investment cost, $M$, one can estimate the profit per unit time, $A$, as (Cook, 1997):

$$
A=D(P-C)-F-M
$$

This calculation is typically done on a quarterly, yearly or multi-year net present value basis. Extending the considerations over multiple time periods is done via the discounted cash flow method (NPV).

Each of the blocks in Fig. 3 represents a body of knowledge and a community of practioners and academics in its own right. Numerous are the arguments that are given (particularly in academia, less in industry) why one cannot or should not consider connecting the six aforementioned domains with simplified models to gain insights into the product realization process. Indeed, one must be careful to apply well grounded methods and appropriately vetted data in such a pursuit. Furthermore, there is no alternative if one wishes to quantitatively answer strategic questions such as the one dealing with platform extent except to rely exclusively on "instinct" and "experience" of senior executives. The methods discussed here are primarily meant to augment current practice and should not supplant sound judgment.

We are primarily interested in a relative comparison of competing platform strategies such as the ones shown in Fig. 2, rather, than in absolute profit forecasting. We will carefully stipulate our assumptions and subject the answers provided by such analysis to sensitivity analysis. The next subsections go into more depth on modeling of the six domains. While alternative methods and references exist, we will only illustrate one method in each of the domains. This will be done in the context of the automotive industry, building upon a comprehensive data set (Appendix A). This data is conveniently reused in Section 5 for the multi-platform problem.

\subsection{Product Architecture}

Product Architecture has been practiced since the industrial revolution, but has only recently emerged as a field of serious research (Meyer \& Rechtin, 2000; Ulrich \& Eppinger 2004). We have found that thinking about and visualizing simultaneously form 
(objects) and function (processes) is one of the main challenges in product architecting. Object Process Methodology (OPM) (Dori, 2002) has emerged as an increasingly popular graphical and formal language for representing product architectures. Figure 4 shows the basic architecture of an automobile. In this single representation we find the structure of the product, decomposed into assemblies (modules), as well as its main value-delivering function (transporting), along with the operands (driver, passengers, cargo), and internal functions. The relationships between design variables, $\mathbf{x}$, and functional attributes, $\mathbf{f}$, can be formally mapped in this way. While the design variables characterize the parts and assemblies, the functional attributes, f, are associated with the internal functions.

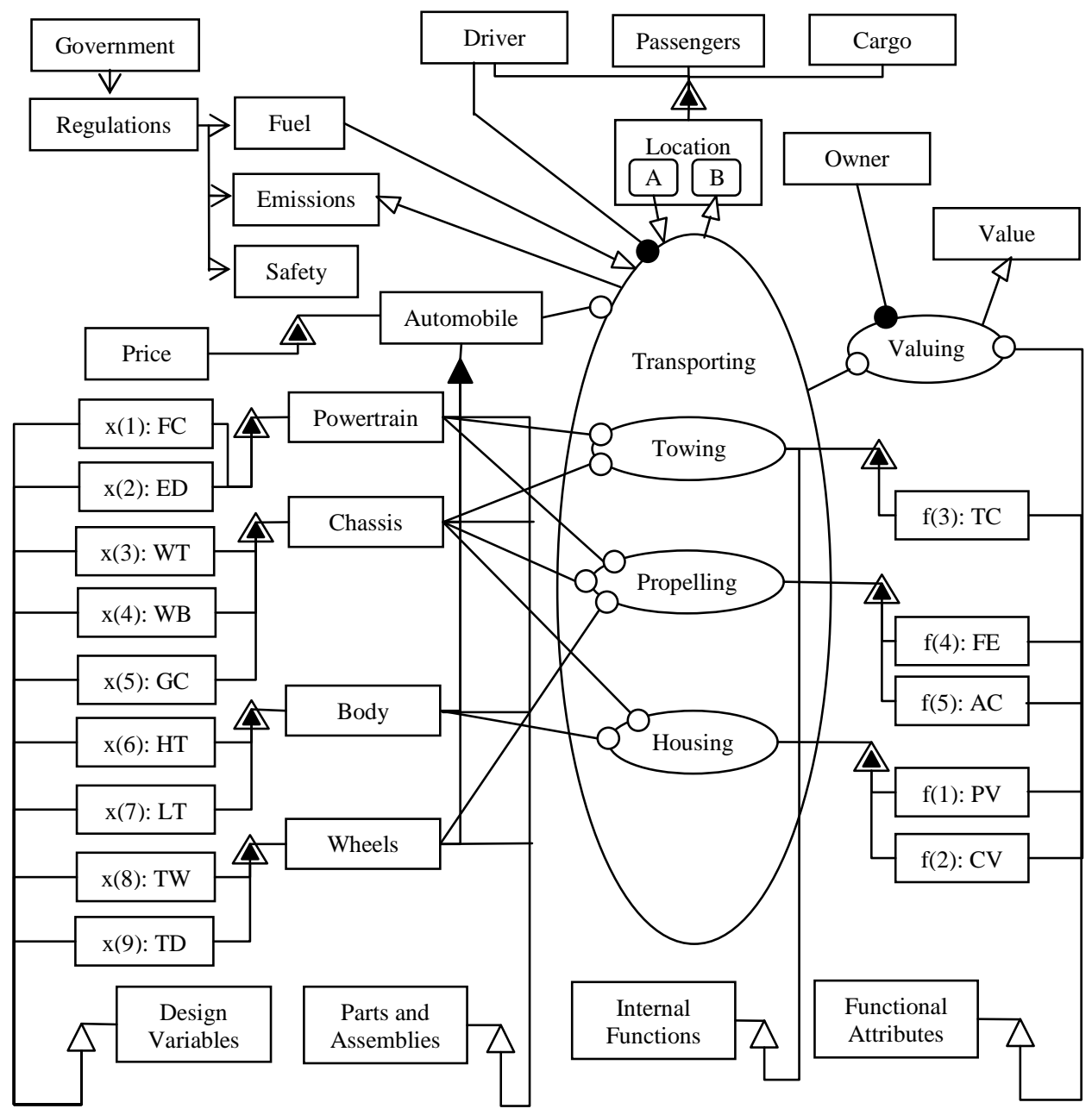

Figure 4: Object Process Diagram (OPD) of a generic automobile. See nomenclature at the end of the chapter for details about design variables and functional attributes. 
A more thorough discussion of the product architecture shown in Figure 4 requires introduction of the object process nomenclature in Figure 5 as well as a simplified visualization of the product system itself (in this case the automobile) in Figure 6.

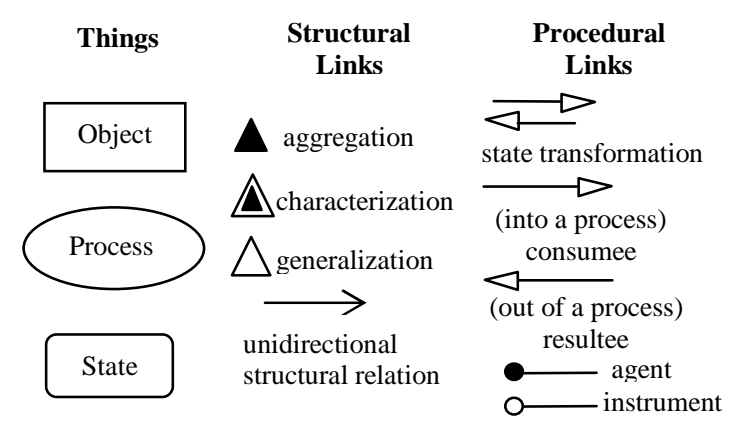

Figure 5: OPM Nomenclature

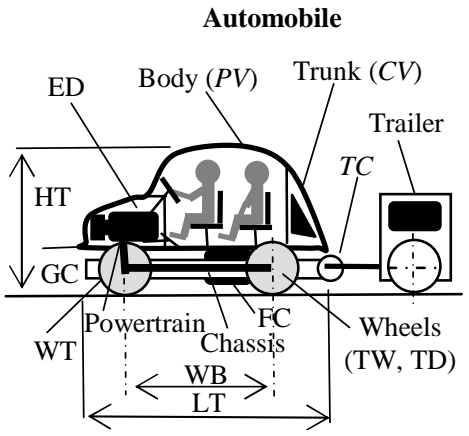

Figure 6: Automobile Schematic

The primary value-delivering function of an automobile is transporting certain numbers of passengers and amounts of cargo comfortably, quickly and economically from location A to B. This has to be done while meeting all government regulations and standards regarding fuel economy, emissions and safety. We call the passengers and cargo the operands of the process, whose attribute state "location" is being transformed from " $\mathrm{A}$ " to " $\mathrm{B}$ ". The driver is both an operand of the process and at the same time the operator (agent) executing the process. The driver can be the owner of the automobile, but this is not necessarily so. The automobile is the main instrument of the process called "transporting".

Moreover, the owner is the individual who made the decision to purchase the automobile at a known price $P$. At the time of the transaction he or she made this decision based on an assessment of the difference between perceived value $V$ and price $P$. The value of the product (from a rational utilitarian point of view) is a continuous quantity which depends on how well the product fulfills its primary, externally delivered function ${ }^{\$}$. The question of "how well" can be quantified via a set of functional attributes, f. In Figure 4, right hand side, we show five important functional attributes of an automobile: passenger volume (PV), cargo volume (CV), towing capacity (TC), fuel economy (FE) and acceleration (AC). There is no claim that this is a complete list of all relevant functional attributes of an automobile. However, the fact that these attributes are readily available in various consumer publications and that they describe concisely the amounts of operand that can be transported or towed by the automobile as well as the (transient) speed and fuel efficiency is compelling.

In fact, if one "zooms" (Dori 2002) into the primary value delivering function of the automobile shown as the large oval labeled "Transporting" in Figure 4, then lower level internal functions are revealed: Propelling, Housing, Towing. Propelling is the ability of the vehicle to roll on a surface as well as to accelerate and decelerate on command. The primary vehicle modules responsible for this process are the powertrain, the chassis and the wheels. The powertrain comprises, among other parts the fuel tank, engine,

\footnotetext{
${ }^{\S}$ We acknowledge that collectors of automobiles potentially have non-utilitarian value functions which are not considered in this work.
} 
transmission, drive shaft and differential. The chassis is made up primarily of the structural underbody (carriage), the braking system as well as the suspension system. The wheels allow the vehicle to roll and transmit the torque generated by the engine to the road. These statements reflect a mapping from internal functions to parts and assemblies. Creating this function-to-form mapping is the primary responsibility of the product architect (Crawley 2001).

The body of the automobile houses the passengers and cargo, thus shielding them from wind and external elements. It also reduces drag and contributes significantly to the external aesthetic appeal of the vehicle (styling). In a "body-on-frame" (BOF) architecture the chassis and body are clearly separated, whereas in a body-frame-integral (BFI) architecture they are more tightly integrated (Whitney et al. 2004). Finally, the towing capacity $(T C)$ of a vehicle is primarily driven by the power of the engine and the ability of the chassis to transmit the towing load from the hitch through the frame and on to the wheels.

It is apparent that the "design variables" of the product characterize the parts and assemblies of the product system. Thus, when making things "common" in product platforming and module reuse it is not sufficient to make the values of design variables common if one intends to actually manufacture common parts and assemblies. In other words, learning curves and economies of scale only materialize by reusing physical components (or software) ${ }^{* *}$. We see from Figure 4 (lower left) and Figure 6 that the powertrain is characterized by the fuel tank capacity (FC) and engine displacement (ED). The chassis is defined by the wheel track $\left(\mathrm{WT}^{\dagger \dagger}\right)$, wheel base (WB) and ground clearance (GC). Overall total length (LT) and height (HT) are associated with the body, which can be of type BOF or BFI, while the wheels are characterized by tire width (TW) and diameter (TD).

Obviously, there will be many more design variables than these nine in a comprehensive automobile realization program. For purposes of our illustrations we will remain with this limited set because: (i) they characterize in major part the four modules in Fig.4 and, (ii) data on these variables is readily available and (iii) they provide enough complexity to yield interesting results. It is understood that a manufacturing firm will have to design and model the product in much more detail. In fact it has been estimated that the bill of materials (BOM) of a typical automobile contains on the order of 10,000 parts, which leads to a drawing tree on the order of

$$
N_{\text {level }}=\left\lceil\frac{\log N_{\text {parts }}}{\log 7}\right\rceil
$$

\footnotetext{
** There is a more subtle notion of architecture with the use of common "hard points", which could be interpreted as a platform even without sharing physical parts. We will take a more traditional view here and assume that platforming implies physical commonality.

${ }^{\dagger}$ We define WT as the front wheel track in this chapter. The difference between front and rear wheel track is usually very small in passenger cars, but can be more significant in trucks.
} 
levels deep. The above equation assumes a 7-tree decomposition-aggregation of the product at each level in accordance with the $7+/-2$ rule imposed by human cognition limits (Miller 1956). Thus, we would expect the drawing tree of an automobile to be about five levels deep. The number of design variables for an individual automobile likely exceeds 100,000 (over 10 design variables per part). Note, however, that the design variables called out in Fig. 4 and 6 are associated with modules rather than individual components. For brevity we will treat these modules as the atomic units of the product. If the front-end question of platform strategy and extent has to be answered for a relatively simple product (drill, walkman...) one may be able to decompose and model the product to its lowest level. For complex products above three levels of decomposition (>300 parts) one generally has to work with a more aggregate representation, as is done here.

It is certainly true that the value of an automobile or any other utilitarian product does not only depend on the functional attributes, f, discussed thus far. The marketing literature refers to "soft" attributes (Cook 1997), i.e. those that cannot be directly predicted by engineering models. Examples of such attributes are: mechanical quality, comfort, styling, mechanical dependability or even the service experience at the dealership. We will discuss the importance of these attributes and how they contribute to value in Section 3.4. The soft attributes have been neglected in Fig. 4 for the sake of clarity.

\subsection{Engineering Performance}

The product architecture is usually an input given to the design engineering team by the product architect(s). This means that the externally-delivered value-generating function as well as the necessary internal functions have been defined and mapped to hardware or software modules and parts to at least two levels of decomposition. It also means that a design vector, $\mathbf{x}$, has been defined, but that specific values for the $x_{i}$ 's are yet unknown.

The main task of design engineers is therefore to determine feasible and perhaps even "optimal" values for the design variables $\mathbf{x}$, subject to lower and upper bounds and other technical constraints. This is done by choosing values for the $x_{i}$ 's such that the functional attributes $\mathbf{f}$ are brought as close to their required targets as possible. These targets are typically defined by (inbound) marketing. Engineers need to also keep track of fixed parameters, p, and dependent variables, y. Examples of fixed parameters are material properties as well as road an atmospheric conditions. Fixed parameters cannot be directly affected by design engineers. Dependent variables, $\mathbf{y}$, are those that depend on design variables or other dependent variables, but which are not directly perceived by the customer and therefore only contribute to product value indirectly. Examples of dependent variables are vehicle curb weight and engine horsepower rating.

In order to establish a mathematical, quantifiable mapping $\mathbf{x}: \mathbf{x} \mapsto \mathbf{f}$ engineers develop models. These models fall into the following three broad categories:

- Empirical models (analogous prototypes)

- Physics-based models (virtual prototypes)

- Actual testable product models (physical prototypes) 
Empirical models are essentially "black box" input/output models that approximate the relationships between $\mathbf{x}$ (inputs) and $\mathbf{f}$ (outputs). The typical methods used are: linear regressions, Neural Networks, Response Surface Models (RSM) and Kriging models (Srivastava et al., 2004). What is required in all cases is an extensive an accurate database of existing (or planned) products with sufficient detail to capture the relationships of interest. An example of such a database is provided in Appendix A for the medium sedan/coupe automotive market segment ('MED') in the United States (Autopro 2002). The database shows the design and dependent variables $\mathbf{x}$, and $\mathbf{y}$, respectively in Table A1. The functional and soft attributes as well as prices and actual sales volumes (=demand) for the 2002 calendar year are in Table A2.

One may gain initial insights into the relationships between $\mathbf{x}$ and $\mathbf{f}$ by producing a set of scatter plots for all pairs of variables $\left\{x_{i}, f_{j}\right\}$, whereby $\mathrm{i}=1,2, . ., n$ and $\mathrm{j}=1,2, . ., m$. In our case we have gathered data for nine design variables, $n=9$, and five functional attributes, $m=5$. The database contains $N=31$ competing models from a number of domestic and foreign brands. Thus, $x_{i, k}$ would be the specific value of the $i$-th design variable for the $k$-th model (product). If, for example, we set $i=4$ and $k=7$, we read the total length (LT) of the Chevrolet Impala as $x_{4,7}=200$ [in] from the database. Appendix B (Fig. B1) shows scatter plots of the design variables $x_{1-9}$ along the rows versus functional attributes $f_{1-5}$ along the columns.

A rather naïve approach to creating an empirical model would be to search for the single design variable, $x_{i}$ that correlates most strongly with a given functional attribute, $f_{j}$ :

$$
\begin{aligned}
& \text { find } i \in\{1,2, \ldots, n\} \\
& \text { such that } \max r^{2} \\
& \text { where } r=\frac{1}{N-1} \sum_{k}\left(\frac{x_{i, k}-\bar{x}_{i}}{s_{x_{i}}}\right)\left(\frac{f_{j, k}-\bar{f}_{j}}{s_{f_{j}}}\right)_{j}
\end{aligned}
$$

where $\bar{x}_{i}$ and $s_{x_{i}}$ are the mean and standard deviation of the $i$-th design variable and $\bar{f}_{i}$ and $s_{f_{j}}$ are the mean and standard deviation of the $j$-th functional attribute, respectively. In this fashion one could create five independent curve fits for each $f_{j}$ as a function of the most strongly correlated single design variable $x_{i}$ :

$$
\hat{f}_{j}\left(x_{i}\right)=a \cdot x_{i}+b
$$

Inspection of Fig. B1 reveals that while some statistical relationships exist, there is much scatter in the data. Some pairs exhibit relatively strong positive correlation such as

\# The prices in Table A2 represent manufacturer recommended sales prices (MSRP) and not actual transaction prices. Actual transaction prices are typically lower than MSRP due to dealer rebates, leasing arrangements and other cash incentives, but reliable information on them is difficult to obtain. 
total length (LT) versus cargo volume (CV) with $r=0.79$ and $r^{2}=0.62$, while others exhibit negative correlation such as engine displacement (ED) versus $0-100 \mathrm{~km} / \mathrm{h}$ acceleration time (AC) with $r=-0.72$ and $r^{2}=0.52$. Yet other combinations appear entirely uncorrelated such as fuel capacity (FC) versus cargo volume (CV) with $r^{2}=0.011$ or ground clearance (GC) versus acceleration time (AC) with $r^{2}=0.0006$. Table 1 shows - for each functional attribute - its strongest regressor, the best linear fit coefficients, $a$ and $b$, as well as $r$ and $r^{2}$ for each fit.

Table 1: Parameters for a univariate empirical model to predict $\mathbf{f}$ from $\mathbf{x}$

\begin{tabular}{ccccccccc}
\hline \hline$j$ & $f(j)$ & $i$ & $x(i)$ & $r$ & $r^{2}$ & $a$ & $b$ & $\mathcal{E}_{R M S, j}$ \\
\hline 1 & PV & 4 & WB & 0.75 & 0.56 & -58.7 & 1.47 & 3.8 \\
2 & CV & 7 & LT & 0.79 & 0.62 & -20.2 & 0.19 & 6.3 \\
3 & TC & 6 & HT & 0.28 & 0.08 & -2831.5 & 72.5 & 23 \\
4 & FE & 2 & ED & -0.68 & 0.45 & 31.4 & -0.003 & 6.3 \\
5 & AC & 2 & ED & -0.72 & 0.52 & 13.7 & -0.002 & 9.4 \\
\hline \hline
\end{tabular}

The functional attributes of the $N$ database entries can be predicted in this fashion. The goodness of the overall empirical model can be assessed via the root mean square (RMS) error of the residuals for the $j$-th attribute:

$$
\varepsilon_{R M S, j}=100 \cdot\left[\frac{1}{N} \sum_{k=1}^{N}\left(\frac{\hat{f}_{j, k}\left(x_{i, k}\right)-f_{j, k}}{\bar{f}_{j}}\right)^{2}\right]^{\frac{1}{2}}
$$

For example, we see that passenger volume is predicted relatively well by wheel base alone (3.8\% error), while towing capacity is predicted poorly ( $23 \%$ error). Across all functional attributes this uncoupled linear model has an average error of $\varepsilon_{\mathrm{RMS}}=9.3 \%$. Figure 7 shows the linear regression for all five functional attributes of interest. This certainly is a crude representation of the mapping from $\mathbf{x}$ to $\mathbf{f}$. The largest problem - aside from the magnitude of the prediction error - is that the coupling within the system is neglected entirely. An example would be that one might choose to increase passenger volume by stretching the car, i.e. increasing the wheel base (WB). This will invariably lead to an increase in curb weight, which would lead to an increase in acceleration time, everything else being held constant. This effect is important when making platforming decisions and it is not captured by the linear regression model where acceleration is a function of engine size alone.
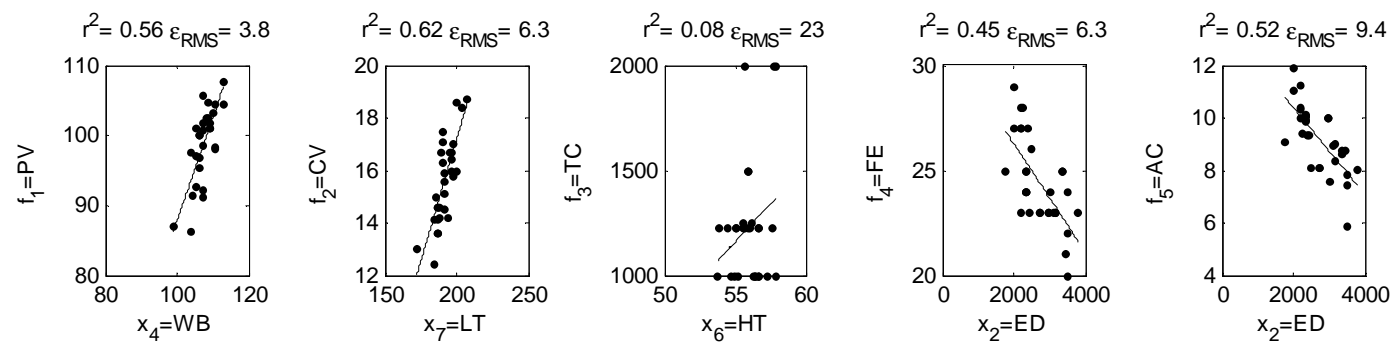

Figure 7: Linear univariate regression model for functional attributes (MED segment) 
To address this deficiency we will construct a simplified response surface model (multivariate polynomial regression) of a vehicle (Srivastava et. al, 2004). We will choose the regressors based on physical insight and we will introduce two independent variables for which data is readily available (curb weight and horsepower rating). The model is developed for one functional attribute at a time.

Passenger Volume: The amount of available space in the passenger compartment (Fig. 6 center) is expected to scale with wheel track (width), wheel base (distance between axles), height and total length. We stipulate the following response surface:

$$
f_{1}=a_{0}+a_{1} x_{3}+a_{2} x_{4}+a_{3} x_{6}+a_{4} x_{7}+a_{5} x_{3} x_{4} x_{6}
$$

The results using optimal least squares coefficients for passenger volume and the other functional attributes are shown in Figure 8.

Cargo Volume: The cargo volume in medium sedans in primarily determined by the trunk space, which in turn depends on the width of the vehicle and the rear overhang (distance from rear axle to rear bumper). For convenience we reuse Equation (6) for cargo volume, but expect to obtain different least squares coefficients $a$.

Towing Capacity: In order to provide a large towing capacity a vehicle needs to have a strong engine (high horsepower rating), a sturdy chassis to sustain the axial loads induced by the trailer, a long wheelbase for directional stability, and a reasonably large curb weight relative to the load. Also, more subtly, frontal area (approximated as width times height) and ground clearance (center of gravity location) are relevant factors. We first approximate the horsepower rating [hp] of the vehicle as a function of engine displacement:

$$
y_{1}=b_{0}+b_{1} x_{2}
$$

Moreover, let curb weight [lbs] be a function of fuel capacity (FC), engine size (ED), width (WT), height (HT) and overall vehicle length (LT):

$$
y_{2}=b_{o}+b_{1} x_{1}+b_{2} x_{2}+b_{3} x_{3}+b_{4} x_{6}+b_{5} x_{7}
$$

Finally, towing capacity is modeled as:

$$
f_{3}=a_{0}+a_{1} y_{1}+a_{2} y_{2}+a_{3} x_{3}+a_{4} x_{4}+a_{5} x_{5}+a_{6} x_{6}
$$

Fuel Economy: It is apparent from Fig. B1 ( $4^{\text {th }}$ column) that vehicles with larger engines have inferior fuel economy. Those vehicles also tend to be heavier. Furthermore, frontal area (width times height) contributes to drag increases even though this can be mitigated by efficient aerodynamic styling. Fuel Economy is therefore approximated as:

$$
f_{4}=a_{o}+a_{1} x_{2}+a_{2} y_{2}+a_{3} x_{3} x_{6}+a_{4} x_{9}
$$


Acceleration: Assuming constant acceleration we can write

$$
\begin{array}{ll} 
& v=\ddot{x} t+v_{0} \text { and } \ddot{x}=F / m \\
\text { with } & P=F \dot{x}=F v \text { and } F=P / v
\end{array}
$$

we obtain the following approximation for acceleration time from $0-100 \mathrm{~km} / \mathrm{h}$ :

$$
t=\frac{v^{2} m}{P}=f_{5}=\left(\frac{100000}{3600}\right)^{2} y_{2} \cdot \frac{0.45}{746 y_{1}}
$$

The correlations between actual functional attributes and those predicted by the RSM model are shown graphically in Fig. 8 with numerical values provided in Table 3 .
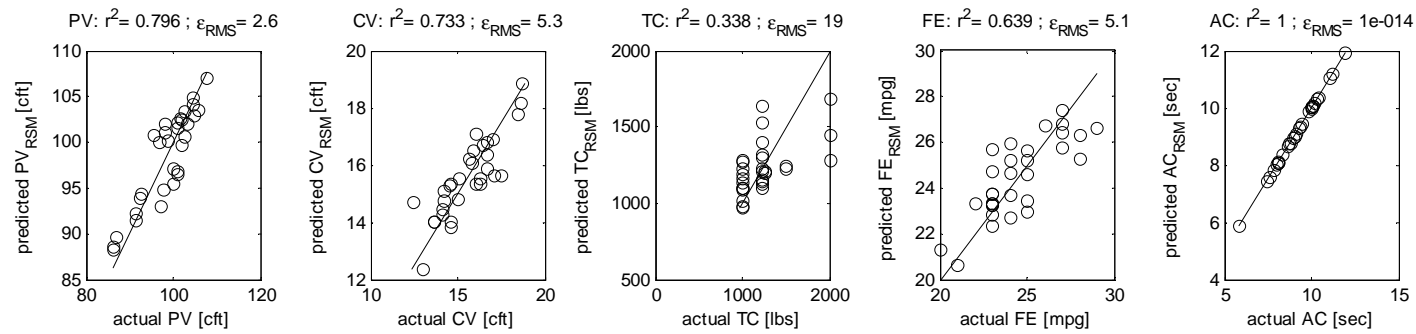

Figure 8: Correlations between RSM prediction and actual vehicle attributes (MED)
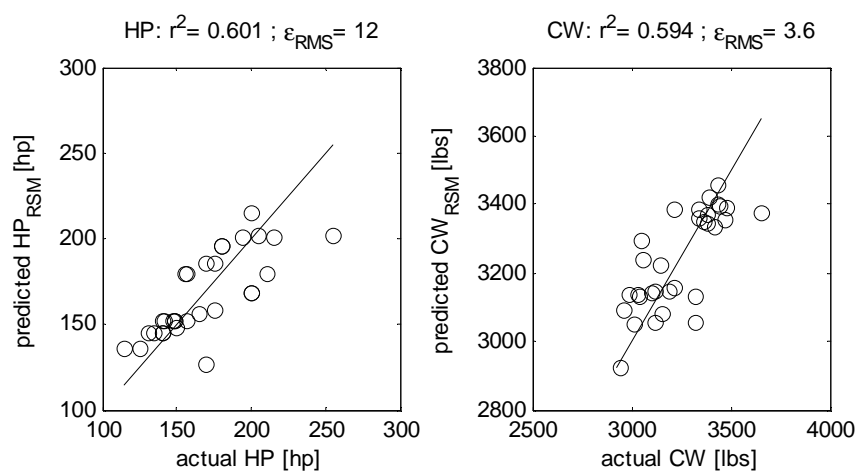

Figure 9: Correlations between RSM prediction and dependent variables (MED)

Table 3: RSM Prediction with $10 \%$ stretched version of a vehicle

\begin{tabular}{l|cl|ccccc}
\hline \hline Vehicle & $\mathrm{x}(4)$ & $\mathrm{x}(7)$ & $\mathrm{f}(1)$ & $\mathrm{f}(2)$ & $\mathrm{f}(3)$ & $\mathrm{f}(4)$ & $\mathrm{f}(5)$ \\
& $\mathrm{WB}$ & LT & $\mathrm{PV}$ & $\mathrm{CV}$ & $\mathrm{TC}$ & $\mathrm{FE}$ & $\mathrm{AC}$ \\
\hline Actual Honda Accord & 105.1 & 186.8 & 92.7 & 13.6 & 1000 & 28 & 9.42 \\
RSM of Honda Accord & 105.1 & 186.8 & 94.5 & 14.0 & 1077 & 25.72 & 9.87 \\
10\% Stretched Version Accord & 115.6 & 205.5 & 107.6 & 16.9 & 1264 & 24.67 & 10.39 \\
\hline \hline
\end{tabular}


We can see from Figure 8 that the predictive accuracy of the RSM is $6.3 \%$ on average, which is better than that of the univariate linear regression model. Stretching the vehicle lengthwise by $10 \%$ increases passenger and cargo volume, but worsens fuel economy and acceleration performance slightly which is what we expect (Table 3). This is primarily due to the increased curb weight. Such a model is useful for conducting platform trade studies.

As mentioned above, one may revert to more detailed physics-based modeling or even to physical prototypes to establish reliable predictions for the functional attributes. For the purposes of this work we will be satisfied with using the response surface models (RSM) developed above. With an average accuracy of 6.3\% they represent sufficiently accurate placeholders for the engineering performance domain block shown in Figure 3.

\subsection{Product Value}

The primary role of the value model is to translate the functional attributes, $\mathbf{f}$, into a scalar quantity called "value", $V$. We accept Cook's (1997) definition of value as an absolute scalar quantity in monetary units, e.g. [\$], which represents the aggregate benefit or worth the consumer derives from a product or service. The perceived net value obtained by the buyer at the time of purchase is $V-P$. The benefit to the seller is $P-C$, where $C$ represents the total cost to manufacture, advertise and distribute the product ${ }^{\S \S}$.

The concept of functional value as a continuous function of the performance attributes of the product is given as:

$$
V\left(f_{1}, f_{2}, \ldots, f_{m}\right)=V_{o} v\left(f_{1}\right) v\left(f_{2}\right) \ldots v\left(f_{m}\right)+\Delta V\left(f_{1}^{\prime}\right)+\ldots+\Delta V\left(f_{k}^{\prime}\right)+\Delta C_{o w n}
$$

where $V_{o}$ is value of a baseline product (typically defined as the "average" product offered in a market segment), $v\left(f_{j}\right)$ 's are the single attribute value curves, $\Delta V\left(f_{k}\right.$ ') are value additions due to product options and $\Delta C_{\text {own }}$ is the change in cost of ownership relative to the baseline product. The value curves take the form:

$$
v\left(f_{j}\right)=\left[\frac{\left(f_{C}-f_{I}\right)^{2}-\left(f_{j}-f_{I}\right)^{2}}{\left(f_{C}-f_{I}\right)^{2}-\left(f_{0}-f_{I}\right)^{2}}\right]^{\gamma}
$$

This quadratic equation is derived from Taguchi's loss function (Cook 1997) such that the value of Eq.(15) goes to zero when $f_{j}$ falls above a critical threshold value, $f_{C}$, for smaller-is-better (SIB) attributes, and asymptotes to a maximum value when $f_{j}$ is below the ideal value, $f_{I}$. An example is acceleration time from $0-100 \mathrm{~km} / \mathrm{h}$. An ideal value of that attribute might be $f_{I}=2$ seconds (similar to Formula 1 race cars), while a critical value might be around $f_{C}=20$ seconds above which it would become dangerous to enter a busy highway. If Eq. (15) goes to zero $\left(f_{j}=f_{C}\right)$ it renders the entire product worthless as shown in Eq. (14), except for the salvage value of the options. If we set $f_{j}=f_{0}$, the

\footnotetext{
$\S \S$ In some countries the sales price, $P$, also contains anticipated expenses for end-of-life
} disposal, but this is not considered here. 
baseline value, then Eq. (15) becomes unity. The value curve is weighted by an exponent, $\gamma$, which expresses the sensitivity of a particular functional attribute relative to others. For our analysis we will ignore the role of product options and cost of ownership and focus on the multiplicative part of Eq. (15). Figure 10 shows sample value curves for our functional attributes, $f_{1-5}$.
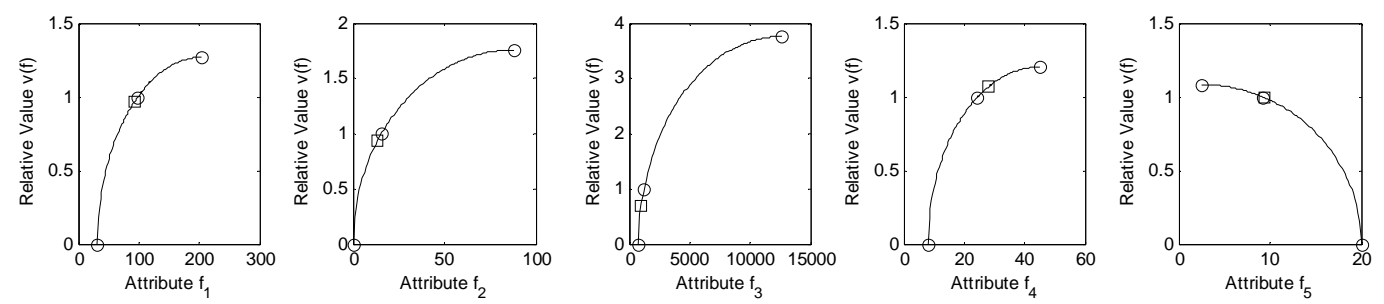

Figure 10: Value curves for $v(f)$ for functional attributes $f_{1}-f_{5}(\gamma=0.5)$. The first four curves are of the type "LIB" (larger-is-better), while the last is "SIB" (smaller-is-better).

The critical and ideal values for each functional attribute are shown in Table 4. These values were found by first determining the minimum and maximum value for each attribute across all 7 market segments considered in this chapter (SML, MED, LRG, SPT, VAN, SUV, TRK) and then going $20 \%$ above and below that extreme value. The baseline values (middle point ' $\mathrm{o}$ ' in the curves in Fig. 10) are chosen as the average values for the MED market segment. Finally, the values of the market leader, Honda Accord, were used as the $f_{i}$ values of interest (square symbols ' ' in Fig.10).

Table 4: Critical, ideal and baseline value for relative value curves

\begin{tabular}{cccccc}
\hline \hline Values & $f(1) P V$ & $f(2) C V$ & $f(3) T C$ & $f(4) F E$ & $f(5) A C$ \\
\hline Type & LIB & LIB & LIB & LIB & SIB \\
Units & [cft] & [cft] & {$[\mathrm{lbs}]$} & {$[\mathrm{mpg}]$} & {$[\mathrm{sec}]$} \\
Critical values $f_{C}$ & 32 & 0 & 800 & 8 & 20 \\
Ideal Values $f_{I}$ & 205 & 88 & 12,600 & 45 & 2.5 \\
Baseline Values $f_{0}$ & 98.5 & 15.6 & 1225 & 24.4 & 9.2 \\
Honda Accord $f_{i}$ & 92.7 & 13.6 & 1000 & 28 & 9.42 \\
\hline \hline
\end{tabular}

Substituting these values into Eq. (15) and then Eq. (14) yields the result that the value of the Honda Accord is 0.66 times the value of the hypothetical baseline (average) product in the market segment. Clearly, this cannot be true, since there must be a reason this particular model leads its segment in terms of sales volume and it doesn't seem to be reflected by our initial value assumptions. There are three potential reasons why this is occurring. First, there are missing product attributes which contribute to value. Second, it is not clear that the weighting factors, $\gamma=0.5$, are correct. Third, there exist factors apart from product attributes such as availability, brand image and intensity of promotion that drive sales. We address the first issue below and the second issue in the next section.

There is ample evidence that "soft attributes" are significant contributors to product value in the automotive industry (Cook 1997). These attributes cannot be directly predicted by engineering analysis, but they can be quantified by customer surveys. We 
introduce the following "soft" attributes, $s_{1-5}$ (J.D. Power 2004), all of which are reported on a scale from 1-5:

1. Mechanical Quality (MQ): score that captures owner-reported problems with the engine, transmission, steering, suspension and braking systems in the first 90 days of ownership.

2. Comfort (CO): score that quantifies features that consumers like and dislike about their vehicles with emphasis on comfort, convenience features and seats.

3. Style (ST): this score is based on how consumers rate the interior and exterior styling of their vehicle, uniqueness of styling, and interior and exterior color choices.

4. Mechanical Dependability (MD): captures owner-reported problems after three years of ownership with the engine, transmission, steering, suspension and braking systems.

5. Service Experience (SE): reflects the quality of the repair and maintenance service consumers received at the dealership. This attribute captures enterprise performance more than direct product performance.

The soft attribute scores for the vehicles in the medium car segment are reported in the last five columns of Table A2. The value curves for the soft attributes, assuming exponential weighting factors of $\gamma=0.5$, are shown in Figure 11.
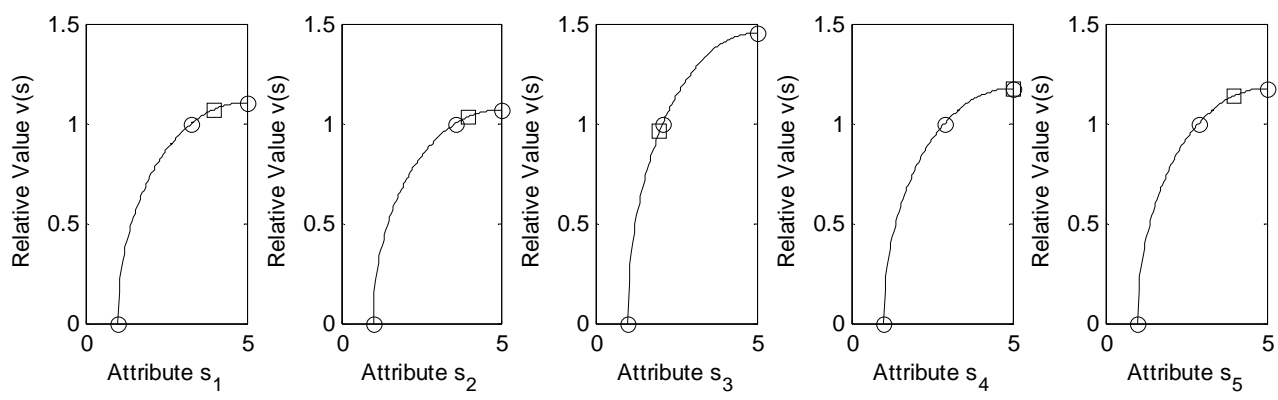

Figure 11: Soft attribute value curves: $s(1)=M Q, s(2)=C O, s(3)=S T, s(4)=M D, s(5)=S E$ with critical values at a score $=1$ and ideal values at score $=5 ; \gamma=0.5$ for all curves.

Again, the average market segment values of soft attributes are used as the baseline, $\mathrm{s}_{\mathrm{o}}=\left[\begin{array}{lllll}3.3 & 3.6 & 2.1 & 2.9 & 2.9\end{array}\right]^{\mathrm{T}}$. Note that the scores for the Honda Accord are shown as the small squares (" ") in Fig. 11. This vehicle achieves a relative value of 1.42 on the soft attributes alone and experts in the automotive industry concur that the strengths of this brand indeed lie in the areas of mechanical quality and dependability.

Cook (1997) suggests that an actual revealed market value, $V_{a}$, can be found, when both price and demand for a product are known. So, we have two kinds of value which must be brought into equivalence: product value as constructed from aggregation of functional and soft attribute constituents and value as revealed by the marketplace via price and demand. The next section focuses on how demand for a product can be predicted as a function of value and price and how this information can be used to automatically (not arbitrarily) find the appropriate weighting factors, $\gamma$. 


\subsection{Market Demand}

Cook's (1997) linear demand model predicts for monopolistic (single product) markets that:

$$
D=K(V-P)
$$

where $D$ is the demand in units sold per unit time, $K$ is the absolute elasticity of demand, $V$ is the product value and $P$ is the sales price. In a competitive market with $i=1,2, \ldots, N$ competitors demand increases, as well as price and value differences introduced by one competitor will affect others. According to Cook's S-Model the demand of the $i$-th product relative to a cartel reference state is captured by the following expression:

$$
D_{i}=K\left[\left(V_{i}-P_{i}\right)-\frac{1}{N} \sum_{j \neq i}\left(V_{j}-P_{j}\right)\right]
$$

where the summation is over all $N$ competitors in the segment. The coefficient $K$ is the negative of the slope of the demand curve at the reference state. The value of the product in the reference state is equal to the price at which demand for the product, as given by the extrapolation of the linear portion of the demand curve through the reference state, goes to zero. The coefficient $K$ should be set to its value at the reference state, namely:

$$
K=E_{1} \frac{D_{R}}{P_{R}} \approx \frac{E_{1} \bar{D}}{\bar{P}}
$$

where $\bar{P}$ is the average price for the segment and $\bar{D}$ is the average demand for competing products. The price elasticity of demand $E_{l}$ is formally defined as:

$$
E_{1} \equiv \frac{-\delta D_{i} / D_{R}}{\delta P_{i} / P_{R}}=\frac{-\delta D_{i} / \bar{D}}{\delta P_{i} / \bar{P}}=\frac{K P_{i}}{D_{i}}
$$

when only product $i$ changes price. We assume this elasticity to be the same for other products in the segment. The change in demand for product $i$ when all products in the segment are increased in price by $\delta P$ gives rise to another price elasticity defined by:

$$
E_{2} \equiv \frac{-\delta D_{i} / D_{i}}{\delta P_{i} / P_{i}}
$$

which is assumed to be independent of the type of product $i$. Because the elasticity $E_{2}$ is independent of the number of competitors, it is more fundamental and is related to $E_{1}$ by:

$$
E_{1}=N E_{2}
$$

Similar to Cook we will only consider the top seven selling vehicles in each segment, and we select $N=7$. In the medium market segment (Table A2) the top seven 
selling vehicles (Honda Accord through Nissan Altima) accounted for only 7/31 (23\%) of the models (variants) available for purchase in 2002, but were responsible for $1.87 / 3.80$ million vehicles (49.4\%) sold in the MED segment. We therefore restrict our considerations of this (and the other market) segment to only the top seven sellers. A frequent difficulty lies in estimating a reliable value of either $E_{2}$ or $K$ without detailed microeconomic data about particular markets. The approach followed here is to set $E_{2}=1$, which yields the result, after substitution into Eq. (21, 18 and 17), that

$$
\bar{V}=\bar{P}\left(\frac{1+E_{2}}{E_{2}}\right)=2
$$

product value is twice the product price, on average. Using the system of $N$ linear equations (17) for demand we can then solve for the "revealed" value in terms of actual demands and prices occurring within a market segment:

$$
V_{i}=P_{i}+\frac{N \bar{P}\left[m_{i}+1\right]}{[N+1] E_{2}}
$$

where

$$
m_{i}=\frac{D_{i}}{\sum_{j=1}^{N} D_{j}}=\frac{D_{i}}{D_{T}}
$$

is the market share of the $i$-th product, i.e. the fraction of demand for product $i$ relative to the total demand for the $N$ competing products in the market segment. Equation (23) and the data in the columns labeled " $D$ " and " $P$ " in Table A2 give us the opportunity to estimate the market revealed value of the $N$ products in this market segment, see Table 5.

Table 5: Market revealed value estimation for medium car segment (MED)

\begin{tabular}{lllcccc}
\hline \hline i & Brand - Model & $\begin{array}{l}D_{i} \\
\text { Table A2 }\end{array}$ & $\begin{array}{l}m_{i} \\
\text { Eq. 24 }\end{array}$ & $\begin{array}{l}P_{i} \\
\text { Table A2 }\end{array}$ & $\begin{array}{l}V_{i} \\
\text { Eq. 23 }\end{array}$ & $V_{i} / P_{i}$ \\
\hline 1 & Honda Accord & 414,718 & 0.22 & 18,890 & 38,547 & 2.041 \\
2 & Toyota Camry & 390,449 & 0.21 & 18,970 & 38,419 & 2.025 \\
3 & Ford Taurus & 353,560 & 0.19 & 19,035 & 38,167 & 2.005 \\
4 & Chevrolet Impala & 208,395 & 0.11 & 20,325 & 38,210 & 1.880 \\
5 & Pontiac Grand Am & 182,046 & 0.10 & 17,135 & 34,794 & 2.031 \\
6 & Chevrolet Malibu & 176,583 & 0.09 & 17,760 & 35,372 & 1.992 \\
7 & Nissan Altima & 148,345 & 0.08 & 16,649 & 34,019 & 2.043 \\
& Average & 267,728 & 0.14 & 18,395 & 36,790 & 2.0 \\
\hline \hline
\end{tabular}

At this point we discussed two different ways to predict product value. Eq. (23) predicts the value of a product as it has been revealed in the marketplace by consumer purchasing patterns. In Eq. (14) on the other hand we stipulated a multiplicative model of product value as an aggregate of functional (and soft) attributes. Both values have to be equivalent. This leads us to solve for the optimal least-squares weighting coefficients, $\gamma$, 
that will minimize the difference between the demand predicted from the aggregate value (Eq. 14) and the demand actually revealed by the market (Eq. 23):

$$
\begin{aligned}
& \text { find } \gamma_{j}^{*}, j=1,2, \ldots, m \\
& \text { s.t. } \min \varepsilon_{R S S}=\sum_{i=1}^{N}\left(D\left(V_{i}\right)_{\text {market }}-D\left(V_{o} v\left(f_{1}\right) \ldots v\left(f_{m}\right)\right)_{\text {predicted }}\right)^{2}
\end{aligned}
$$

This procedure was followed for the MED market segment and the resulting optimal weighting coefficients are shown in Table 6.

Table 6: Optimal weighting coefficients $\gamma^{*}$ to predict product value and demand ${ }^{* * *}$

\begin{tabular}{|c|c|c|c|c|c|c|c|c|c|}
\hline$f_{l}=P V$ & $f_{2}=C V$ & $f_{3}=T C$ & $f_{4}=F E$ & $f_{5}=A C$ & $s_{1}=M Q$ & $s_{2}=C O$ & $s_{3}=S T$ & $s_{4}=M D$ & $s_{5}=S E$ \\
\hline 0.560 & 0.030 & 0.033 & 0.030 & 0.03 & 0.261 & 0.061 & 0.134 & 0.030 & 0.641 \\
\hline
\end{tabular}

Figure 12 shows the resulting value curves for the MED car segment, where the optimal $\gamma^{*}$ 's from Table 6 and the critical, ideal and baseline values from Table 4 have been used. These are indicative of consumer preferences in this particular segment. The magnitude of the exponents alone is somewhat misleading in understanding the 'most important' attributes, since the shapes of the value curves are also influenced by the positions of the critical, ideal and baseline values for each attribute.
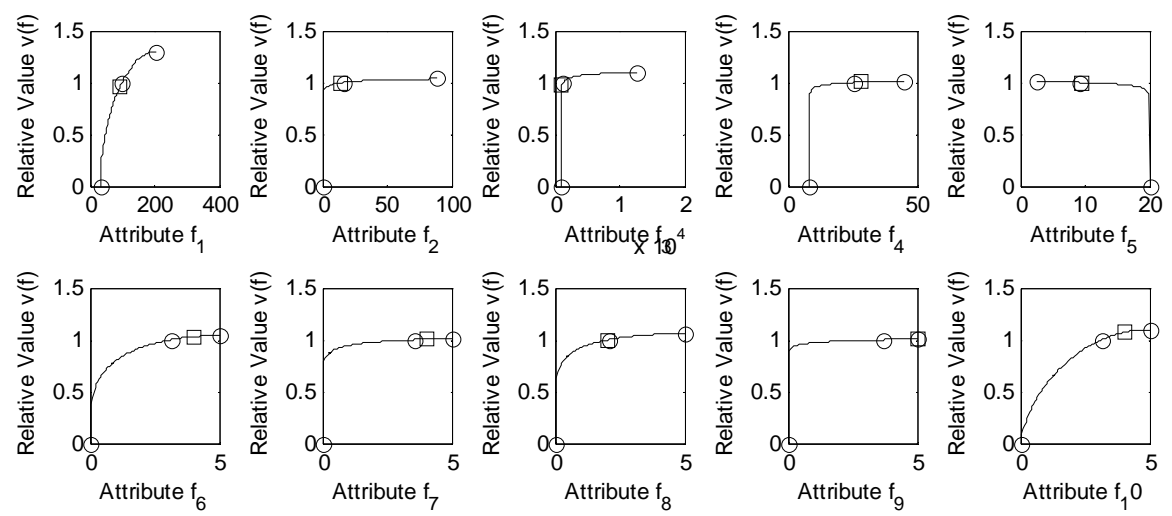

Figure 12: Market-revealed value curves for all $m=10$ attributes in the 'MED' segment. Attributes $s_{1-5}$ are treated as $f_{6-10}$ during analysis

From Table 6 and Fig. 12 we learn that this segment appears to be very sensitive to passenger volume $\left(f_{l}\right)$, initial mechanical quality $\left(f_{6}\right)$ and service experience $\left(f_{10}\right)$ and somewhat sensitive to styling and comfort. The demand predicted by the value model and the actual demand match quite closely as is seen in Fig. 13. Because some exponents are at their lower bounds, a small correction factor for $K=K^{*} K_{\text {corr }}$, with $K_{\text {corr }}=1.046$ has to be introduced to maintain a constant size of the predicted market segment.

\footnotetext{
*** The lower bound for $\gamma$ was set to 0.03 , the upper bound to 1.0. These bounds correspond to lower and upper values of the exponent $\gamma$ found in Cook (1997, Table 5.3).
} 


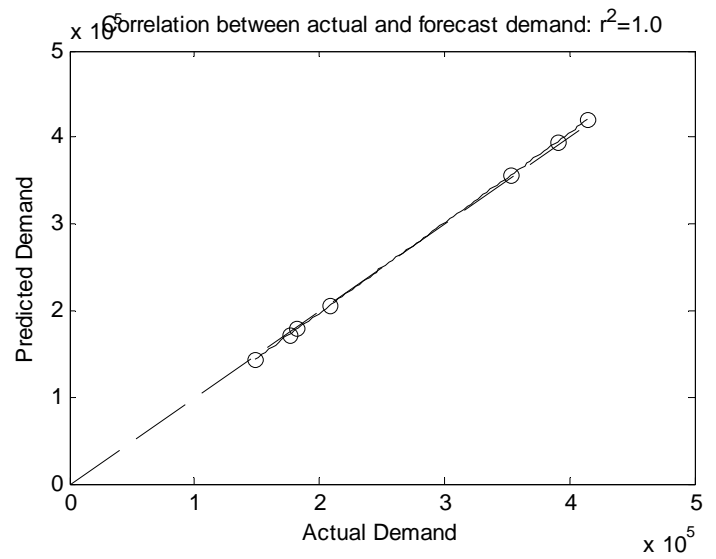

Figure 13: Correlation between forecasted and actual demand for top 7 sellers in MED market segment. Demand prediction made with Eq. (17).

The benefit of the value and demand models is that demand can be predicted as a function of perturbations to the functional and soft attributes. Such changes are introduced indirectly via manipulation of the design variables associated with various product design and platform strategies. These physical changes in the product can now be propagated to estimate changes in demand across multiple market segments. This obviates the need for introducing artificial concepts such as "acceptable performance losses" when comparing platform design alternatives.

\subsection{Manufacturing Cost}

The main function of the manufacturing cost model is to estimate the total cost of manufacturing the product. The total cost of manufacturing $D_{i}$ instances of the $i$-th product in a unit time period is given as (Cook 1997):

$$
C T_{i}=D_{i} C_{i}+F_{i}
$$

where $D_{i}$ is the demand (sales volume) discussed in the previous section, $C_{i}$ is the variable (per unit) cost, referred to as "marginal costs", and $F_{i}$ is the fixed cost. The relationship between operating revenues, $P_{i} D_{i}$, total cost $C T_{i}$ and operating earnings (pre-tax profit), $A_{i}$, is shown in Figure 14. 


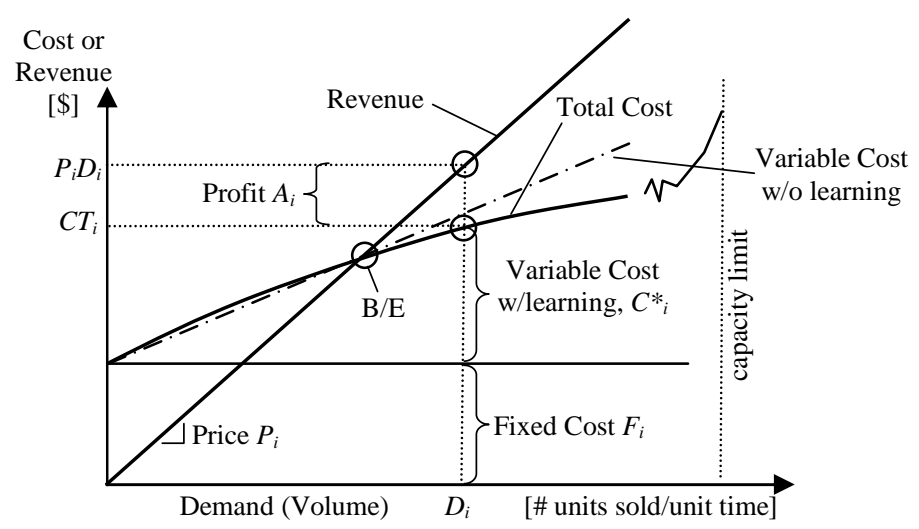

Figure 14: B/E Diagram: Cost and Revenue as a function of demand (volume)

We can rewrite the operating profit of the $i$-th product from Eq. (1) as:

$$
A_{i}=\alpha_{i} P_{i} D_{i}
$$

where $\alpha$ is the operating (profit) margin. Thus, total costs are estimated as:

$$
C T_{i}=\left[1-\alpha_{i}\right] P_{i} D_{i}
$$

The next important piece of information is the operating leverage, $\phi$, i.e. the relative split between fixed and variable costs, see Eq. (26). We can approximate this as:

$$
F_{i}=\phi_{i} C T_{i}=\phi_{i}\left[1-\alpha_{i}\right] P_{i} D_{i}
$$

Since building cars and trucks is a capital intensive business we expect the average operating leverage to be at least $\phi_{i}=0.3 .{ }^{\dagger \dagger}$ This yields an estimate of the total variable cost at the nominal production operating point, $D_{i}$ of:

$$
C_{i}=\left(1-\phi_{i}\right)\left(1-\alpha_{i}\right) P_{i} D_{i}
$$

Similarly, the per-unit variable cost is:

$$
c_{i}=\left(1-\phi_{i}\right)\left(1-\alpha_{i}\right) P_{i}
$$

Modeling of the variable costs of a product is typically done by one of the following three methods:

\footnotetext{
${ }^{\dagger \dagger}$ Later we will discuss a sensitivity analysis with respect to profit margin $\alpha$, operating leverage $\phi$, and the cost breakdown coefficients, $\beta_{i k}$.
} 
1. Bottom-up process oriented: model individual fabrication \& assembly steps

2. Cost-estimation-relationships (CER's): fit regression curves to historical cost data of precursor products/systems.

3. Costing-by-analogy: take a known product and its cost as a reference baseline and calculate differential costs with respect that that baseline by adjusting for changes in design variables or product options.

We will pursue the third approach, assuming knowledge of $\alpha$ and $\phi$. Moreover, an important reason for platforming is the promise of being able to achieve a significant learning curve. This learning curve manifests itself as progressively decreasing variable per unit cost, shown by the flattening total cost curve in Fig. 14. Thus, we rewrite the variable cost per unit as a function of production volume as:

$$
c_{i}\left(D_{i}\right)=c_{t f u, i} \cdot D_{i}^{B}=\sum_{k=1}^{n_{\bmod }} c_{t f u, i, k} \cdot D_{i}^{B}
$$

where

$$
B=\log S / \log 2
$$

$S$ is the learning curve factor. The factor $S$ represents the average relative variable cost of a unit, each time the number of production units is doubled. Typically, $S$ is on the order of 0.9 for (parts) fabrication, 0.75 for assembly and 0.98 for material expenditures. Overall one often assumes $0.8<S<1.0$. The variable $c_{t f u i, i, k}$ is the theoretical first unit cost of the $k$-th module of the $i$-th product, where $n_{\text {mod }}$ is the number of modules in the product. One can first solve for the theoretical first unit cost of the entire product by equating (32) and (31) and rearranging

$$
c_{t f u, i}=c_{i}\left(D_{i}\right) \cdot D_{i}^{-B}=\left(1-\phi_{i}\right)\left(1-\alpha_{i}\right) P_{i} D_{i}^{-B}
$$

The variable cost breakdown for the main modules of a vehicle (Table 7) is assumed as follows:

$$
c_{t f u, i, k}=\beta_{i k} c_{t f u, i}, \quad \text { where } k=1,2, \ldots, n_{\text {mod }}
$$

Table 7: Assumed cost breakdown coefficients $\beta_{i k}$

\begin{tabular}{lllllll}
\hline \hline$i$ & Market & $\beta_{i 1}$ & $\beta_{i 2}$ & $\beta_{i 3}$ & $\beta_{i 4}$ & Total \\
& Segment & Powertrain & Chassis & Body & Wheels & \\
\hline 1 & SMP & 0.35 & 0.30 & 0.25 & 0.10 & 1.0 \\
2 & MED & 0.30 & 0.35 & 0.30 & 0.05 & 1.0 \\
3 & LGP & 0.25 & 0.35 & 0.35 & 0.05 & 1.0 \\
4 & SPT & 0.40 & 0.30 & 0.22 & 0.08 & 1.0 \\
5 & SUV & 0.30 & 0.35 & 0.30 & 0.05 & 1.0 \\
6 & VAN & 0.25 & 0.30 & 0.40 & 0.05 & 1.0 \\
7 & TRK & 0.35 & 0.4 & 0.20 & 0.05 & 1.0 \\
\hline \hline
\end{tabular}


An estimate of the average, theoretical first unit costs of each module, in each product, in each market segment can therefore be obtained - using market segment averages - as:

$$
c_{t f u, i, k}=\bar{\beta}_{i k}(1-\bar{\phi})(1-\bar{\alpha}) \bar{P} D_{i}^{-B}, \text { where } k=1,2, \ldots, n_{\mathrm{mod}}
$$

For the medium passenger car segment (Appendix A) with $D_{i}=267,728$ (market segment average sales volume among top 7 selling vehicles) we arrive at:

Table 8: Theoretical first unit cost calculations for 'MED' market segment $(i=2)$

\begin{tabular}{cccccccc}
\hline \hline$S$ & $\bar{\phi}$ & $\bar{\alpha}$ & $\bar{P}$ & $\begin{array}{l}c_{t f u, 2,1} \\
\text { Powertrain }\end{array}$ & $\begin{array}{l}c_{t f u, 2,2} \\
\text { Chassis }\end{array}$ & $\begin{array}{l}c_{t f u, 2,3} \\
\text { Body }\end{array}$ & $\begin{array}{c}c_{t f u, 2,4} \\
\text { Wheels }\end{array}$ \\
\hline 0.95 & 0.3 & 0.1 & $\$ 18,395$ & $\$ 8,766$ & $\$ 10,227$ & $\$ 8,766$ & $\$ 1,461$ \\
\hline \hline
\end{tabular}

This results in a theoretical first unit cost per vehicle of $c_{t f u, 2}=\$ 29,221$, according to Eq. (34). The cost breakdown shown in Table 8 is only valid for an average product whose design variables all have the average settings of the first $N=7$ competitors. Because variable cost is not only affected by production quantity, but also by other factors such as the amount of material used, we scale the theoretical first unit cost of each module with respect to the average design variable settings as follows:

$$
\begin{aligned}
& c_{t f u, i, 1}^{\prime}=c_{t f u, i, 1} \cdot \frac{x_{i, 2}}{\bar{x}_{2}} \\
& c_{t f u, i, 2}^{\prime}=c_{t f u, i, 2} \cdot \frac{x_{i, 3} x_{i, 4}}{\bar{x}_{3} \bar{x}_{4}} \\
& c_{t f u, i, 3}^{\prime}=c_{t f u, i, 3} \cdot \frac{x_{i, 3} x_{i, 6} x_{i, 7}}{\bar{x}_{3} \bar{x}_{6} \bar{x}_{7}} \\
& c_{t f u, i, 4}^{\prime}=c_{t f u, i, 4} \cdot \frac{x_{i, 8} x_{i, 9}}{\bar{x}_{8} \bar{x}_{9}}
\end{aligned}
$$

The variable powertrain cost scales linearly with engine displacement ${ }^{+*}$, the variable chassis cost scales linearly with footprint area (WB times WT), the variable cost of manufacturing the body scales with enveloping volume and the variable wheel costs scale with wheel diameter and tire width.

Given this information, one may now estimate the total variable cost of a vehicle product $\left(C_{i}^{\prime}\right)$, given its market segment $(i)$, production volume $\left(D_{i}\right)$ and design variable settings $\left(\mathbf{x}_{\mathrm{i}}\right)$ as:

$\$+$ In a more detailed cost model one would want to capture whether or not the engine is naturally aspirated or boosted (turbo charged). 


$$
C_{i}^{\prime}\left(D_{i}, \mathbf{x}_{i}\right)=c_{t f u, i}^{\prime} \cdot D_{i}^{B}=\sum_{k=1}^{n_{\text {mod }}} c_{t f u, i, k}^{\prime}\left(\mathbf{x}_{i}\right) \cdot D_{i}^{B}
$$

The total cost of production is then:

$$
C T_{i}=D_{i} C^{\prime}{ }_{i}+F_{i}
$$

The per unit variable costs, $C$, fixed costs, $F$, operating profit, $A$, and margin, $\alpha$, were simulated for the $N=7$ competitors in the MED market segment in this fashion (Table 9). The same assumptions were made as in Table 8. In particular we assume that a hypothetical manufacturer who sells an average vehicle and achieves an average sales volume in this segment achieves a profit margin of $\alpha=10 \%$, which includes the $2 \%$ average margin of the dealers in the sales network (Harris 2002) ${ }^{\S \S \S}$.

Table 9: Manufacturing cost simulation for 'MED' market segment

\begin{tabular}{llcccccc}
\hline \hline $\mathrm{j}$ & Model & $P[\$]$ & $D$ & $\begin{array}{c}F \\
{\left[10^{9} \$\right]}\end{array}$ & $C^{\prime}[\$]$ & $A\left[10^{9} \$\right]$ & $\alpha$ \\
\hline 1 & Honda Accord & 18,890 & 414,718 & 1.3297 & 10,407 & 2.1883 & 0.28 \\
2 & Toyota Camry & 18,970 & 390,449 & 1.3297 & 10,861 & 1.8363 & 0.25 \\
3 & Ford Taurus & 19,035 & 353,560 & 1.3297 & 11,984 & 1.1632 & 0.17 \\
4 & Chevrolet Impala & 20,325 & 208,395 & 1.3297 & 13,323 & 0.1485 & 0.04 \\
5 & Pontiac Grand Am & 17,135 & 182,046 & 1.3297 & 10,834 & -0.1826 & -0.06 \\
6 & Chevrolet Malibu & 17,760 & 176,583 & 1.3297 & 12,276 & -0.3614 & -0.12 \\
7 & Nissan Altima & 16,649 & 148,345 & 1.3297 & 12,086 & -0.6528 & -0.26 \\
\hline \hline
\end{tabular}

The results predict that 4 out of 7 models will generate a profit, under the assumption that the fixed cost, $F$, is the same to produce each model ${ }^{* * * *}$. Also, we see that a large production volume (assuming the product is actually sold at price $P$ ) is beneficial in two ways: (i) it generates significant revenue, and (ii) the per-unit cost $C^{\prime}$ is lowered due to the learning curve, Eq. (32). Figure 15 shows where the 7 competitors fall on the break-even diagram corresponding to the hypothetical average manufacturer. It is interesting to note that the actual average profit margin, $\alpha$, among the seven models is only $4.2 \%$, which is lower than the $10 \%$ stipulated for an average manufacturer. This difference is explained by the non-linearity introduced through the learning curve and the uneven distribution of sales volumes in the market segment.

\footnotetext{
$\$ \S$ Recent profit margins in the automotive industry have fallen significantly below $10 \%$. ${ }^{* * * * *}$ The fixed cost is mainly due to recurring, but volume independent charges related to manufacturing plants, non-hourly labor as well as equipment, tooling and facilities.
} 


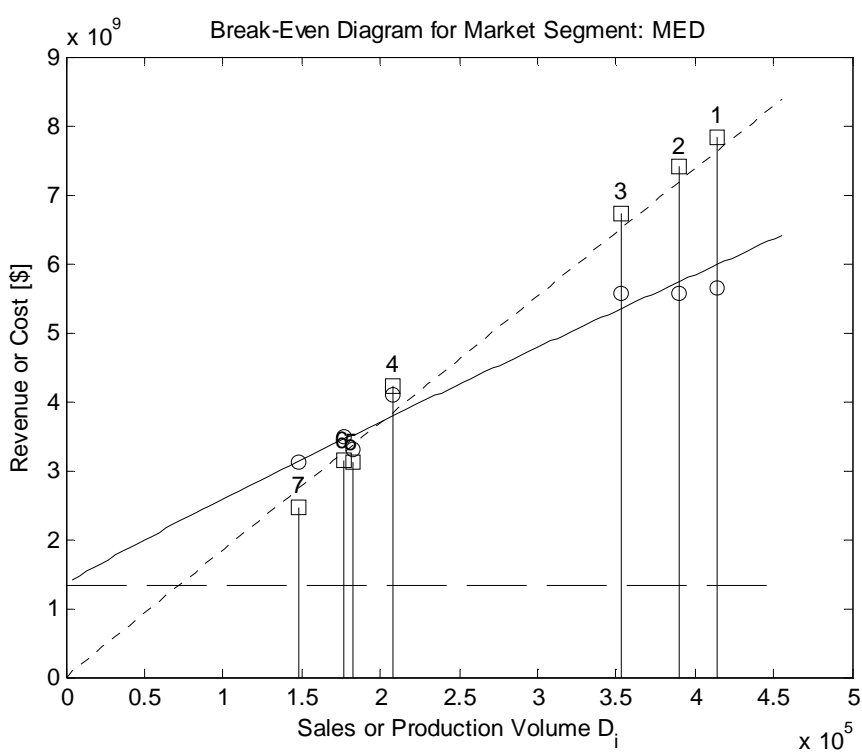

Figure 15: B/E diagram for medium car segment: ' '= revenue, 'o'= cost. Model numbers (1-7) correspond to the top seven selling vehicles shown in Table A2

\subsection{Investment Finance}

In order to compare different platforming strategies with each other one must take into account capital investments, costs (both fixed and variable) as well revenues from sales. The typical cash flow profile of a generic product lifecycle is shown in Figure 16.

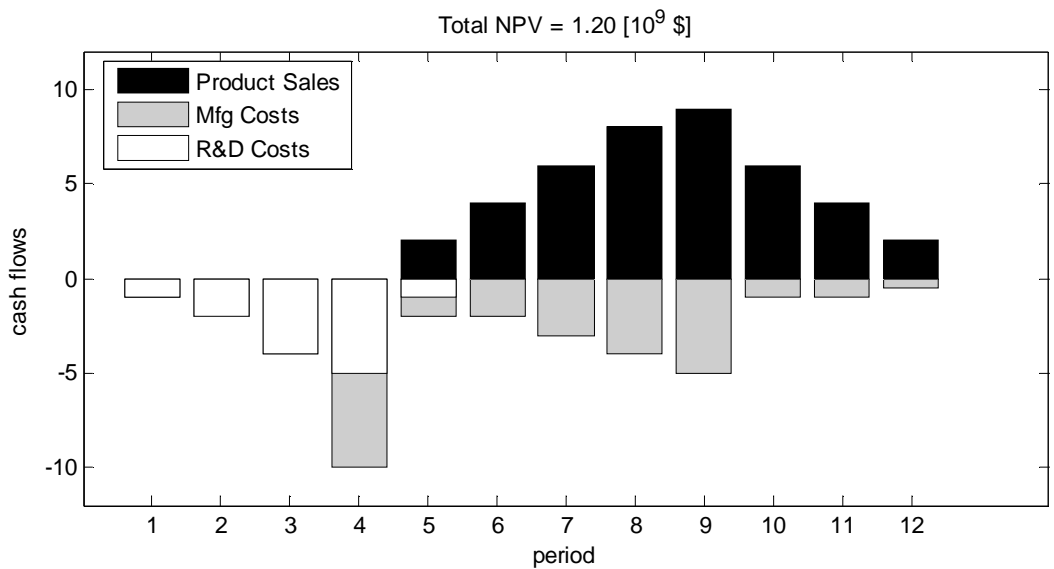

Figure 16: Typical product cash flow profile over a 12-year lifecycle, units [\$B]. Product launch is assumed to occur in year 5 , with tooling being procured in year 4 . 
A number of periods, $N_{R D}$, is spent developing the product, which includes component and module design, integration, prototype manufacturing as well as laboratory and field testing. It used to take at least 48 months $\left(N_{R D}=4\right)$ to develop a completely new vehicle in the automotive industry, but this number has been dropping to below 36 months and is approaching 24 months thanks to more efficient CAD/CAE/CAM processes, increasing design reuse and platforming. Typical $R \& D$ budgets of automotive companies, $M_{R D}$, range between $2-6 \%$ of yearly sales revenues.

After product launch, the sales revenues from a product, $D_{i} P_{i}$, usually ramp up at some rate, peak after a number of periods and then start to decline as the product gradually loses ground to newly introduced competitors. Manufacturing costs are incurred for tooling and plant equipment, fixed expenditures as well as variable costs such as labor and material. The variable costs can be affected by the learning curves (Eq. 32). It is usually advantageous to separate out the investment required in upkeep and modernization of plants and equipment, $M_{P}$, as a percentage of annual sales. As we will see later, the development of one or multiple product platforms can be seen as such an investment, whose primary target is to lower variable costs.

The annual forecasted profit for the $j$-th year and the $i$-th product can then be estimated as:

$$
A_{i, j}=D_{i, j}\left[P_{i, j}-C^{\prime}{ }_{i, j}\right]-F_{i, j}-M_{R D, i, j}-M_{P, i, j}
$$

where demand $D_{i, j}$ is predicted from Eq.(17), price $P_{\mathrm{i}, \mathrm{j}}$ is chosen as a free variable based on pricing strategy, the variable costs $C^{\prime}{ }_{i, j}$ are computed using Eq. (38), fixed costs, $F_{i, j}$, are estimated from Eq. (29) and R\&D costs, $M_{R D, i, j}$, and plant investment costs, $M_{P, i, j}$, are percentages of sales (e.g. 5\% for R\&D). We assume $N_{R D}$ periods for development and $N_{P}$ periods of production. The merits of a particular product realization program can be assessed by its net present value (NPV), sometimes also referred to as net present worth (Cook 1997):

$$
\Pi_{i}=\sum_{j=1}^{N_{R D}+N_{P}} \frac{A_{i, j}}{(1+r)^{j}}
$$

where $r$ is the discount rate which captures the time value of money, at a minimum the risk-free interest rate. ${ }^{1+\dagger}$ The NPV analysis is the final step in the product framework shown in Fig. 3. The predictions for yearly profit, $A_{i, j}$, operating margin $\alpha_{j}$ and the expected NPV, $\Pi i$, of new investments (new product development, new platforms...) are the ultimate decision metric of the for-profit firm and its shareholders.

An NPV analysis was conducted for the market leader in the medium ('MED') passenger car segment and the resulting (non-discounted) cash flow profile is shown in Figure 17. From a comparison of Fig.16 and Fig.17 one can see that a number of simplifying assumptions were made. The majority of these relate to ignoring (unknown) future market dynamics:

H† An example of a "risk free interest rate" is the interest yielded by U.S. government bonds for the period equivalent to the total duration of the project or product lifecycle. 
- The R\&D expenditure profile is flat and the total $\mathrm{R} \& \mathrm{D}$ budget is equivalent to $M_{R D}=m_{R D} N_{P} P_{i} D_{i}$, spread out over $N_{R D}=3$ years.

- $\quad$ The sales volume $D_{i}$ predicted by Eq. (17) remains valid for the $N_{P}=8$ years of production, which implies time invariance of prices $(P)$, customer preferences $(\gamma)$, and technologies $(f(x))$.

- $\quad$ The fixed costs, $F_{i}$, and variable costs, $C^{\prime}{ }_{i}$, are also assumed to be time invariant.

We accept these assumptions for now and will discuss the potential impact of market dynamics later. The simulated cash flow profile for the market leader of the ('MED') segment is shown in Fig. 17, predicting a NPV of $\$ 5.62$ billion over 11 years.

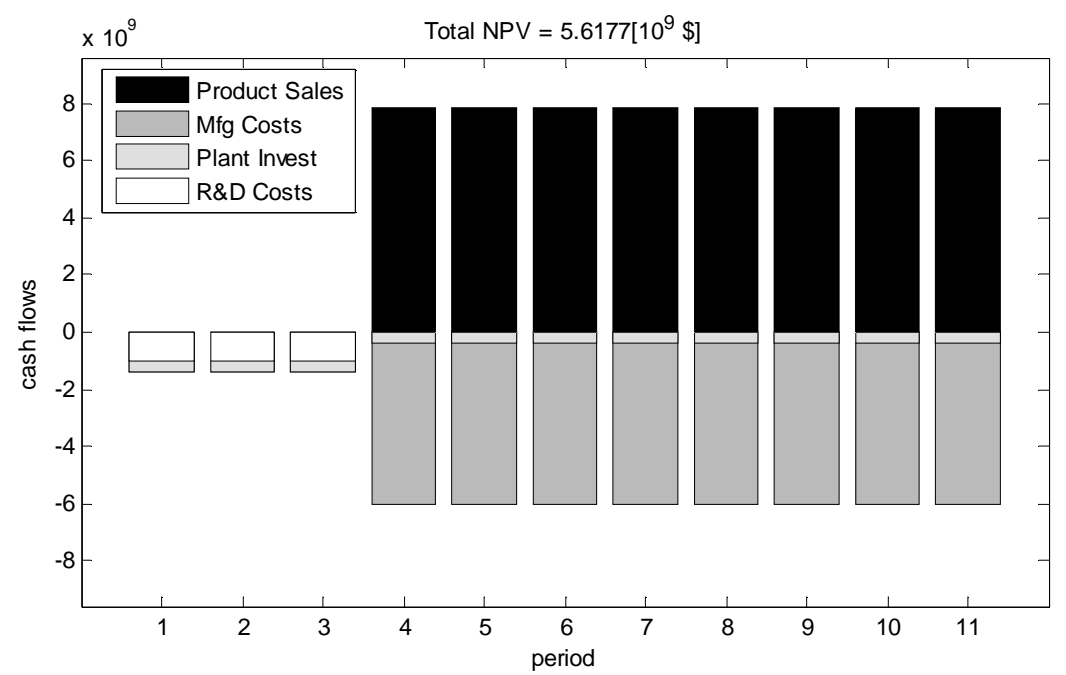

Figure 17: Hypothetical NPV analysis for market leader (see Appendix A) with $N_{R D}=3$, $N_{P}=8, r=0.06, m_{R D}=0.049$ (PSA 2004), $m_{P}=0.068$ (PSA 2004) and sales and manufacturing cost estimates shown in Table 9.

\subsection{Modeling Accuracy}

With the six sub-models of our modeling framework (Fig. 3) completed, we can assess its accuracy by benchmarking against the $N=7$ bestselling products of the MED passenger car market. For each product we input the actual design vector, $\mathbf{x}$, and predict the functional attributes, $\mathbf{f}$, the product value, $V_{i}$, demand, $D_{i}$, profit, $A_{i}$, and operating margin, $\alpha_{i}$. Prediction errors according to the root-mean square (RMS) metric defined in Eq. (5) can be used as a measure of accuracy (Table 10).

Table 10: Model predictions and accuracy for 'MED' segment

\begin{tabular}{llccccccc}
\hline \hline $\mathrm{j}$ & Model & $P[\$]$ & $\begin{array}{c}f: \varepsilon_{R M S} \\
(\%)\end{array}$ & $\begin{array}{c}D \\
\text { actual }\end{array}$ & $\begin{array}{c}D \\
\text { predict }\end{array}$ & $\begin{array}{c}m_{D_{D}}: \\
(\%)\end{array}$ & $\begin{array}{c}A \\
{\left[10^{9} \$\right]}\end{array}$ & $\alpha$ \\
\hline 1 & Honda Accord & 18,890 & 3.41 & 414,718 & 517,397 & 0.35 & 2.1883 & 0.28 \\
2 & Toyota Camry & 18,970 & 13.95 & 390,449 & 298,486 & -7.87 & 1.8363 & 0.25 \\
3 & Ford Taurus & 19,035 & 2.28 & 353,560 & 301,825 & -5.75 & 1.1632 & 0.17 \\
\hline \hline
\end{tabular}




\begin{tabular}{llccccccc}
\hline \hline 4 & Chevrolet Impala & 20,325 & 12.23 & 208,395 & 315,252 & 2.58 & 0.1485 & 0.04 \\
5 & Pontiac Gd Am & 17,135 & 0.13 & 182,046 & 217,998 & -0.24 & -0.1826 & -0.06 \\
6 & Chevrolet Malibu & 17,760 & 19.70 & 176,583 & 374,956 & 6.87 & -0.3614 & -0.12 \\
7 & Nissan Altima & 16,649 & 23.06 & 148,345 & 275,882 & 4.07 & -0.6528 & -0.26 \\
& Average & 18,395 & 10.68 & 267728 & 328828 & 3.96 & 0.591 & 0.043 \\
\hline \hline
\end{tabular}

We see that, on average, the model predicts functional attributes within $10.68 \%$ for the first $N=7$ competitors in this market segment, given only knowledge of the design vector, $\mathbf{x}$, for each vehicle. This confirms that the engineering performance model developed in Section 3.3, using response surface models (RSM), is accurate within 6$11 \%$. The largest error is incurred in predicting towing capacity (TC), but Fig. 12 shows that value is relatively insensitive to this functional attribute, at least in the MED market segment. The demand, $D$, in terms of actual numbers and predictions is shown in columns 5 and 6 of Table 10, respectively. Note that total market size is not preserved when we add up the predictions in column 6 , because the vehicles were evaluated using Eq. (17) one-at-a-time and not concurrently. The corresponding prediction errors in \% market share, $m_{i}$, see Eq. (24), are shown in column 7. While errors in predicting the absolute number of units sold per unit time can be substantial, we find that market share is predicted with an average accuracy of 3.96\% for this market segment. It is difficult to compare predictions of profit on a model-by-model basis because most firms only present aggregate data in their annual reports and don't disclose profit numbers for individual vehicles, either for accounting or tactical reasons. We will therefore proceed with this model for further analysis.

\subsection{Single Product Optimization}

The question one might care to answer at this point is the following: What is the design of the "optimal" vehicle in the MED car market segment that will theoretically maximize NPV? The designers and managers of a single vehicle (without platforming) can choose the following quantities, assuming that the vehicle architecture (Fig. 4) remains fixed::

- Design variables: $x_{i, I^{-}} x_{i, 9}$

- Soft attributes: $s_{i, 1^{-}} s_{i, 5}$

- Price: $P_{i}$

The single vehicle design optimization problem for $N P V$ maximization then becomes:

$$
\begin{array}{ll}
\max & \prod_{i}\left(\mathbf{x}, \mathbf{s}, P_{i}\right) \\
\text { s.t. } & x_{i, j, L} \leq x_{i, j} \leq x_{i, j, U} \quad \mathrm{j}=1,2, \ldots, 9 \\
& 1 \leq s_{i, j} \leq 5 \mathrm{j}=1,2, \ldots, 5 \\
& P_{\min , i} \leq P_{i} \leq P_{\max , i}
\end{array}
$$


As upper and lower bounds of the design variables we choose the minimum and maximum values encountered in the $(N=7)$ market segment (Table A1). The price is also bounded below and above by the maximum and minimum price encountered in the market segment. These restrictions are necessary because, as stated above, the value and demand models are only valid in the vicinity of the cartel reference state (Cook 1997). Furthermore, it is assumed that the product we are designing is introduced as a direct competitor to the other $N=7$ products, which remain fixed at their current settings.

We solve the optimization problem, Eq. (42) using a gradient-based sequential quadratic programming (SQP) algorithm ${ }^{*}$. The algorithm is provided with the settings of the current market leader as the starting point (Table 11).

Table 11: Single vehicle design (initial guess - top), optimal design (bottom) Initial guess

\begin{tabular}{|c|c|c|c|c|}
\hline $\mathrm{Po}=\$ 18,890$ & $\mathrm{Xo}(1)=17.1 \mathrm{~g}$ & $\mathrm{Xo}(2)=2254 \mathrm{ccm}$ & $\mathrm{Xo}(3)=61,2 ”$ & $\mathrm{Xo}(4)=105.1^{\prime \prime}$ \\
\hline $\mathrm{Xo}(5)=3.9^{\prime \prime}$ & $\mathrm{Xo}(6)=54.9 "$ & $\mathrm{Xo}(7)=186.8$ & $\mathrm{Xo}(8)=195 \mathrm{~mm}$ & $\mathrm{Xo}(9)=15^{\prime \prime}$ \\
\hline $\operatorname{So}(1)=4$ & $\operatorname{So}(2)=4$ & $\operatorname{So}(3)=2$ & $\operatorname{So}(4)=5$ & $\operatorname{So}(5)=4$ \\
\hline \multicolumn{5}{|c|}{ Optimal design } \\
\hline $\mathrm{P}^{*}=\$ 20,325$ & $X^{*}(1)=\mathbf{2 0 . 0} \mathbf{g}$ & $X^{*}(2)=2189 \mathrm{ccm}$ & $X^{*}(3)=\mathbf{5 9 . 0}$ & $\mathrm{X}^{*}(4)=105.1^{\prime \prime}$ \\
\hline$X^{*}(5)=3.9 "$ & $X *(6)=57.9 "$ & $X^{*}(7)=\mathbf{2 0 0}$ & $\mathrm{X}^{*}(8)=195 \mathrm{~mm}$ & $X^{*}(9)=15 ”$ \\
\hline $\mathrm{S} *(1)=\mathbf{5}$ & $\mathrm{S} *(2)=\mathbf{5}$ & $\mathrm{S} *(3)=5$ & $\mathrm{~S} *(4)=5$ & $\mathrm{~S} *(5)=5$ \\
\hline
\end{tabular}

The differences between the "optimal" design ${ }^{\S \S \S}$ (bottom) and the current market leader (top) are highlighted in bold in Table 11. First, as expected, all the soft attributes are set to their highest level since there is nothing in the model that would trade against this change. The optimized design has a somewhat larger fuel capacity (FC), smaller engine (ED), smaller wheel track (WT), but is higher (HT) and longer (LT) than the current leader. These changes can be explained by the strong sensitivity of the medium segment to passenger volume (see Table 6) relative to the other functional attributes. Without bounds on the design variables the optimizer would attempt to further increase the gap between value and price in Eq. (17) by increasing the size of the vehicle further. This is prevented by the bounds, as otherwise the vehicle specifications would start to place it in the next larger car segment. This hypothetical vehicle is predicted to achieve an NPV of $\Pi^{*}=60.8 \$ \mathrm{~B}$ over 11 years compared to an estimated NPV of $\Pi_{O}=9.2$ $\$ \mathrm{~B}$ for the initial guess over the same period. How can this large difference be explained?

Table 12 shows a comparison of the initial vehicle and the optimized vehicle in terms of functional attributes, $\mathbf{f}$, and value constituents, $\mathbf{v}$.

Table 12: Comparison of initial vehicle design $\left(\mathbf{x}_{\mathbf{0}}\right)$ and optimized design $\left(\mathbf{x}^{*}\right)$

\begin{tabular}{ccccccccccc}
\hline \hline & $f_{1}=P V$ & $f_{2}=C V$ & $f_{3}=T C$ & $f_{4}=F E$ & $f_{5}=A C$ & $s_{1}=M Q$ & $s_{2}=C O$ & $s_{3}=S T$ & $s_{4}=M D$ & $s_{5}=S E$ \\
\hline$\gamma_{i}$ & 0.560 & 0.030 & 0.033 & 0.030 & 0.03 & 0.261 & 0.061 & 0.134 & 0.030 & 0.641 \\
\hline $\mathbf{f}_{\mathbf{o}}$ & 94.4 & 14.0 & 1077.1 & 25.7 & 9.9 & 4 & 4 & 2 & 5 & 4 \\
$\mathbf{v}_{\mathbf{0}}$ & 0.966 & 0.996 & 0.987 & 1.000 & 0.999 & 1.029 & 1.003 & 0.997 & 1.002 & 1.071 \\
\hline
\end{tabular}

$\$+$ MATLAB V7.0.1 (R14) is used as the modeling environment, with the function fmincon serving as the SQP constrained optimization algorithm.

${ }_{\S \S \S}$ Due to non-convexity of the problem we cannot claim global optimality. 


\begin{tabular}{ccccccccccc}
\hline $\mathbf{f}^{*}$ & 110.1 & 19.1 & 1882.9 & 25.0 & 10.7 & 5 & 5 & 5 & 5 & 5 \\
$\mathbf{v}^{*}$ & 1.061 & 1.004 & 1.032 & 0.999 & 0.997 & 1.040 & 1.005 & 1.058 & 1.002 & 1.100 \\
\hline \hline
\end{tabular}

The optimized vehicle achieves a value of 1.334 , relative to the market segment average, resulting in a value of $V^{*}=\$ 49,078$ and a predicted sales volume of $D^{*}=1.628$ million vehicles. The current market leader on the other hand achieves a relative value of 1.047 with an absolute value of $V_{o}=\$ 38,520$ and a predicted sales volume of $D_{o}=0.517$ million units. Moreover, we notice that both the soft attributes (1.218 vs. 1.103) as well as the functional attributes (1.095 vs. 0.949) contribute to the relative value increase of the optimized vehicle relative to the initial guess. While it might not be realistic to expect a 5-point J.D. Power rating in all the soft attributes in practice, we will allow for this possibility during subsequent platform design and optimization.

\section{SINGLE PLATFORM OPTIMIZATION}

In the previous section we have discussed the single product framework shown in Fig.3, developed the underlying mathematics for each of the six sub-models, and exercised the framework with help of an automotive database (Appendix A) for the medium passenger car market segment. So far, however, we have taken only a single product view. A manufacturing firm wanting to satisfy a larger number of customers whose preferences (both in terms of functional and soft attributes) do not cluster tightly will attempt to satisfy demand with a family of products. Therefore, we will first develop an understanding of clustering of design variables, functional attributes as well as valuevs-price for all seven segments of the automotive market.

Next, we will ask the question how this variety of preferences might be satisfied by a new entrant who wishes to compete with exactly one model in each market segment. There are two fundamental strategies in this respect. First, one may want to treat each product independently of the others such that its characteristics and manufacturing processes may be uniquely tailored. Second, one may build them from one (or more) platforms by reusing common modules or scaling the product in one or more physical or functional dimensions. We will first establish a baseline by "optimally" designing a new product for each market segment, without platforming. Next, we will select one of the modules as the platform and optimize the product family that is based on it.

\subsection{Multiple Market Segment View}

A database of six additional market segments each with its top selling, $\mathrm{N}=7$, competitors has been developed. Table $\mathrm{C} 1$ in Appendix $\mathrm{C}$ shows an overview of average, minimum and maximum values for the design variables and dependent variables in each market segment. Table 13 shows demand elasticity, total market segment size, average price and average value for the top seven vehicles in the given market segment.

Table 13: Comparison of seven market segments (only top 7 products)

\begin{tabular}{cccccc}
\hline \hline $\mathrm{j}$ & $\begin{array}{c}\text { Market } \\
\text { Segment }\end{array}$ & $\begin{array}{c}K \\
\text { elasticity }\end{array}$ & $\begin{array}{c}D_{T} \\
\text { actual }\end{array}$ & $\overline{\bar{P}}[\$]$ & $\overline{\bar{V}}[\$]$ \\
\hline 1 & SML & 113.99 & $1,459,000$ & 12,769 & 25,539 \\
\hline \hline
\end{tabular}




\begin{tabular}{lccrrr}
\hline \hline 2 & MED & 106.60 & $1,874,096$ & 18,395 & 36,790 \\
3 & LRG & 19.09 & 632,894 & 29,928 & 59,856 \\
4 & SPT & 10.46 & 382,473 & 21,330 & 42,661 \\
5 & VAN & 39.97 & 918,193 & 23,197 & 46,394 \\
6 & SUV & 60.05 & $1,488,058$ & 26,492 & 52,983 \\
7 & TRK & 133.06 & $2,778,964$ & 16,587 & 33,173 \\
\hline \hline
\end{tabular}

The value-vs.-price position of the top seven selling vehicles in each segment is shown in Fig. 18. It is interesting to note that the small sedans (SML) are all clustered together in the lower left corner, followed by the medium sedans (MED) along the diagonal to the upper right. The large sedans are typically in the $\$ 22,000-\$ 30,000$ price range with the exception of two outliers (Cadillac Deville, Lincoln Towncar) in the upper right of Fig.18. These two vehicles would probably be broken out into a luxury sedan segment if the market segmentation were done at a finer level of granularity. The outlier in the sports segment is the Chevrolet Corvette. The seven top-selling SUVs don't cluster nicely but span a relatively large price-value range. The (pickup) trucks fill the gap between the small and medium sedans in the price-value space.

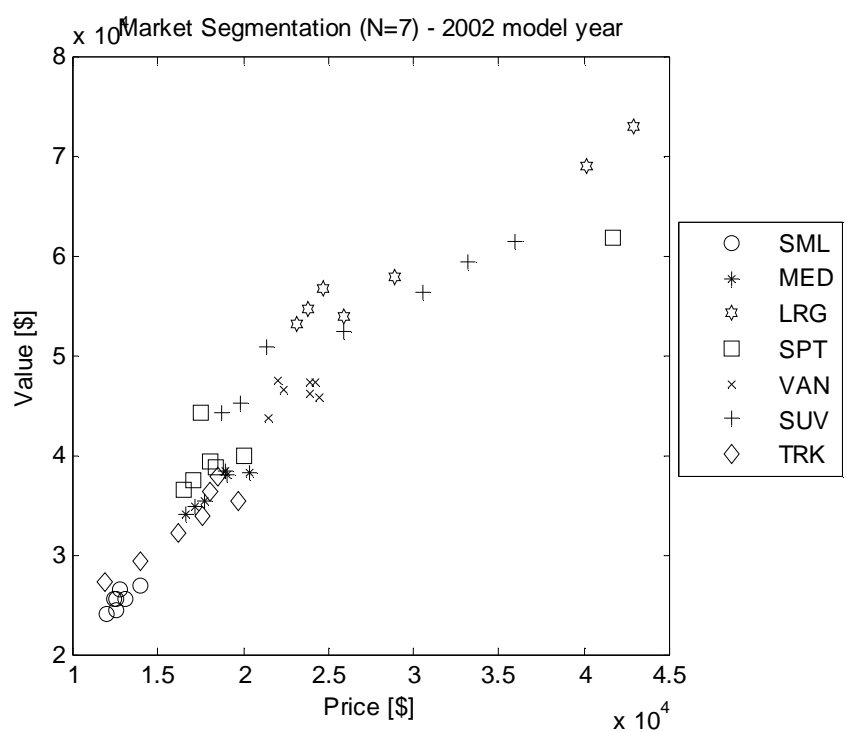

Figure 18: Position of seven top-selling vehicles in seven market segments

How many platforms would be needed to span the space shown in Fig.18 if the manufacturer decided to compete with one model in each market segment? The first step in answering this question is to optimize a vehicle for each segment (as was done in Subsection 3.9) without any platforming commonality constraints. 


\subsection{Baseline Case 1: No Platforming}

This subsection establishes a baseline by computing the "optimal" vehicle design that maximizes NPV (Eq. 41) in each market segment, independently. This optimization is done by assuming that all design variables can be chosen independently of other market segments, constrained only by the bounds given within each segment (Table C1). This therefore reflects the "null platform" case. Or said otherwise, each product is its own platform $(p=m)$ and there is no explicit reuse between segments.

Table 14 shows the initial product family (current market leaders) on top and optimized product family on the bottom. Simulated Annealing (SA) was used as the optimization algorithm $^{* * * * *}$. The bounds on the design variables $\mathbf{x}$ were set by the minimum and maximum occurrences in the product database for each market segment (Table C1).

Table 14: Initial (o) versus optimized product variants without platforming

\begin{tabular}{|c|c|c|c|c|c|}
\hline & Segment & $P[\$]$ & $V[\$]$ & $D$ [units] & $N P V[\$ \mathrm{~B}]$ \\
\hline \multirow{8}{*}{ 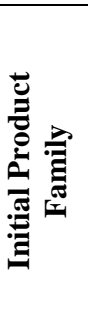 } & $\mathrm{SML}_{\mathrm{o}}$ & 12,810 & 26,389 & 354,650 & 1.92 \\
\hline & $\mathrm{MED}_{\mathrm{o}}$ & 18,890 & 42,531 & $1,006,100$ & 26.98 \\
\hline & $\mathrm{LRG}_{\mathrm{o}}$ & 24,660 & 63,162 & 391,120 & 7.13 \\
\hline & $\mathrm{SPT}_{\mathrm{o}}$ & 17,475 & 72,194 & 440,810 & 6.21 \\
\hline & $\mathrm{VAN}_{\mathrm{o}}$ & 21,980 & 48,743 & 304,490 & 5.03 \\
\hline & $\mathrm{SUV}_{\mathrm{o}}$ & 21,355 & 71,430 & $2,307,000$ & 61.04 \\
\hline & $\mathrm{TRK}_{\mathrm{o}}$ & 18,540 & 58,564 & $3,993,200$ & 133.69 \\
\hline & Total & & & $8,797,370$ & 242.0 \\
\hline \multirow{8}{*}{ 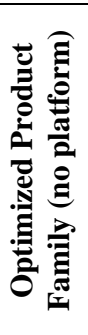 } & SML* & 12,609 & 27,781 & 562,217 & 6.04 \\
\hline & MED* & 20,270 & 49,047 & $1,631,753$ & 57.68 \\
\hline & LRG* & 38,043 & 80,795 & 483,815 & 40.83 \\
\hline & SPT* & 32,768 & 72,974 & 267,309 & 22.24 \\
\hline & VAN* & 22,907 & 51,707 & 397,535 & 9.79 \\
\hline & SUV* & 35,658 & 103,215 & $3,506,711$ & 325.21 \\
\hline & TRK* & 19,569 & 75,294 & $6,380,642$ & 303.01 \\
\hline & Total & & & $13,229,982$ & \begin{tabular}{|l|l|}
764.8 \\
\end{tabular} \\
\hline
\end{tabular}

The optimization suggests that a new market entrant could hypothetically develop a new product family that exceeds the sales volume (D) and net present value (NPV) of the product family comprised of the current market leaders. The optimized product family (without platforming) is predicted to achieve a sales volume of 13.2 million vehicles versus 8.8 million vehicles for the current family, an increase of 50.3\%. The NPV over an 11 year program life (Fig.17) increases from an estimated current 242.00 \$B to 764.8 \$B $(+241.4 \%)$.

How could this be achieved? There are three quantities that the firm can affect directly in tailoring their product offerings: (1) the design variables for each vehicle, $\mathbf{x}_{\mathrm{i}}$,

${ }^{* * * * *}$ The optimal MED vehicle settings in Table 11 and those in Table $15\left(6^{\text {th }}\right.$ row from bottom) are slightly different because SQP was used in the former case, while SA was the optimizer used in the latter case. 
the values of the soft attributes, $\mathbf{s}_{\mathrm{i}}$, and the price, $P_{\mathrm{i}}$. From Table 14 we see that the optimizer chose to lower the price for one vehicle (SML), while increasing the price moderately for some vehicles (MED, VAN, TRK) and significantly for others. In most cases these changes are justified by also increasing the value of the vehicle. All soft attributes, $\mathbf{s}_{\mathrm{i}}$, have been set to their maximum value of 5 . Recall that the optimizer attempts to maximize the NPV for each vehicle, independently of all other vehicles, based on Eq. (41). This equation takes into account changes throughout the product framework (Fig.3) that affect the bottom line (NPV).

Table 15 shows how the optimizer chose to perturb the design of each vehicle from the initial guess to the optimized design. In some cases a vehicle was downsized (e.g. SML car) in terms of its wheelbase (WB) and wheel track (WT) in other cases the vehicle was significantly stretched (e.g. SUV). These changes can only be understood by considering the values of the $\gamma$-weighting factors for each vehicle in each market segment. A detailed discussion of these weighting factors is possible, but beyond the scope of this chapter.

Table 15: Original vehicle designs (top), optimized designs (bottom)

\begin{tabular}{|c|c|c|c|c|c|c|c|c|c|c|}
\hline \multirow{3}{*}{\multicolumn{2}{|c|}{ Segment }} & $\mathbf{x}(\mathbf{1})$ & $\mathbf{x}(2)$ & $\mathbf{x}(\mathbf{3})$ & $\mathbf{x ( 4 )}$ & $\mathbf{x ( 5 )}$ & $\mathbf{x}(\mathbf{6})$ & $x(7)$ & $\mathbf{x}(\mathbf{8})$ & $\mathbf{x}(\mathbf{9})$ \\
\hline & & FC & ED & WT" & WB & GC & HT & LT & TW & TD \\
\hline & & {$[\mathrm{g}]$} & {$[\mathrm{ccm}]$} & [in] & [in] & [in] & [in] & [in] & {$[\mathrm{mm}]$} & [in] \\
\hline \multirow{7}{*}{ 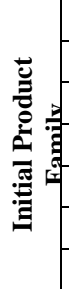 } & $\mathrm{SML}_{\mathrm{o}}$ & 13.2 & 1668 & 57.9 & 103.1 & 5.27 & 55.1 & 174.7 & 185 & 14 \\
\hline & $\mathrm{MED}_{\mathrm{o}}$ & 17.1 & 2254 & 61.2 & 105.1 & 3.90 & 54.9 & 186.8 & 195 & 15 \\
\hline & $\mathrm{LRG}_{\mathrm{o}}$ & 18.5 & 3790 & 62.3 & 112.2 & 5.40 & 57.0 & 200.0 & 215 & 15 \\
\hline & $\mathrm{SPT}_{\mathrm{o}}$ & 15.7 & 3802 & 60.2 & 101.3 & 4.30 & 53.1 & 183.2 & 225 & 16 \\
\hline & $\mathrm{VAN}_{\mathrm{o}}$ & 20.0 & 3301 & 63.0 & 119.3 & 5.60 & 68.9 & 200.5 & 215 & 15 \\
\hline & $\mathrm{SUV}_{\mathrm{o}}$ & 16.8 & 4011 & 58.5 & 101.8 & 6.70 & 68.4 & 180.4 & 235 & 16 \\
\hline & $\mathrm{TRK}_{\mathrm{o}}$ & 25.0 & 4196 & 65.4 & 119.9 & 7.30 & 72.7 & 206.9 & 255 & 16 \\
\hline \multirow{7}{*}{ 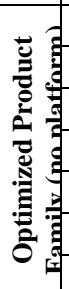 } & SML* & 14.1 & 1720 & 58.8 & 97.0 & 4.9 & 53.0 & 168.1 & 186 & 14 \\
\hline & MED* & 20.3 & 2421 & 59.0 & 106.1 & 4.9 & 57.9 & 200.3 & 212 & 15 \\
\hline & LRG* $^{*}$ & 19.2 & 3283 & 61.1 & 108.1 & 5.2 & 56.7 & 210.5 & 214 & 16 \\
\hline & SPT* & 14.5 & 3106 & 60.6 & 104.8 & 6.3 & 49.7 & 191.5 & 200 & 15 \\
\hline & VAN* & 20.0 & 3076 & 66.5 & 118.0 & 7.3 & 69.1 & 196.9 & 212 & 15 \\
\hline & SUV* & 15.0 & 2679 & 62.0 & 129.5 & 7.7 & 68.2 & 173.0 & 236 & 15 \\
\hline & TRK* & 24.8 & 3504 & 62.4 & 104.3 & 6.7 & 67.0 & 207.4 & 236 & 16 \\
\hline
\end{tabular}

As we can see from Table 15, each vehicle has its own, freely chosen design variables and is not constrained by platforming in any way. It is interesting to note that the engine was downsized for most vehicles (except SML and MED), presumably due to the benefits of better fuel economy. We will take the values of the design variables in the bottom half of Table 15 as the baseline product family, without platforming. The financial performance of this product family is estimated at an aggregate NPVof \$B 764.8 over 11 years.

\subsection{Case 2: Single Platform Optimization}

The question posed here is whether the use of a product platform could potentially improve the financial performance of the product family. If this could be achieved it 
would primarily be via lowering of the variable costs associated with the module which is chosen as the platform, see Eq.(38). The improvement in variable costs would be captured via the manufacturing learning curve, Eq.(32). Thus, with the designation of one module as the product platform, which is shared by at least two products, the estimation of variable costs for the product family has to be revised accordingly:

$$
C_{\text {fam }}=\sum_{i=1}^{m} D_{i} \cdot c_{t f u, p l a f f o r m} \cdot\left[\sum_{i=1}^{m} D_{i}\right]^{B}+\sum_{i=1}^{m} D_{i} \sum_{k=1}^{n_{\text {mod }}-1} c_{t f u, i, k} \cdot D_{i}^{B}
$$

In Eq. (38b) the first term is the variable cost contributed by the platform, which is used by all variants. The learning curve benefits occur from summation of the production volumes over all $m$ variants that reuse the platform. This is one of the main mechanisms by which the use of platforms can lower the cost of the entire product family. The second term in Eq. (38b) is contributed by all the other unique (non-common) modules which are not reused between variants.

The second mechanism by which product platforms lower manufacturing cost is by allowing multiple variants to be produced from the same platform on the same assembly line. Figure 19 shows how three different models follow each other on the same assembly line.

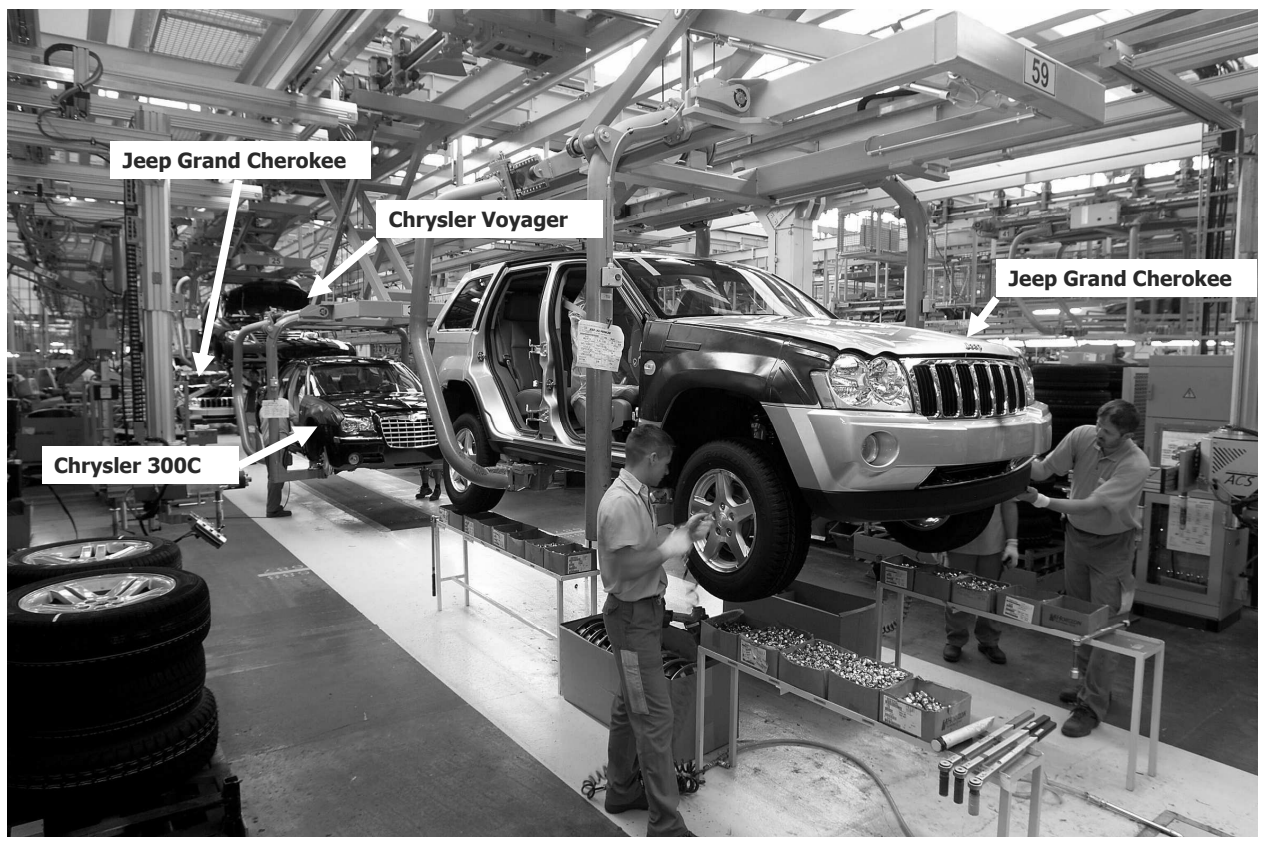

Figure 19: Three models on one assembly line (Daimler-Chrysler, courtesy: Magna)

This effect is captured in our model, by adjusting the fixed costs from their original formulation for the $i$-th product: 


$$
F_{i}=\phi_{i} C T_{i}=\phi_{i}\left[1-\alpha_{i}\right] P_{i} D_{i}
$$

to a formulation where the fixed cost is estimated for the entire product family.

$$
F_{\text {fam }}=\left\lceil\sum_{i=1}^{m} D_{i} / D_{\text {plant }}\right\rceil \cdot \phi_{\text {fam }}[1-\bar{\alpha}] \bar{P} D_{\text {plant }}
$$

Here the first term (in the ceiling function) estimates the number of manufacturing plants that are needed to produce the entire product family by diving the total production volume over all $m$ variants by the plant capacity, $D_{\text {plant }}$, and rounding up to the next integer. Here we assume that a vehicle manufacturing plant has a yearly capacity of 250,000 vehicles. The fixed cost per plant are estimated via the average revenue generated by the plant at full capacity, $\bar{P} D_{\text {plant }}$, the average profit margin, $\bar{\alpha}$, and the operating leverage for the product family, $\phi_{\text {fam }}$.

Which module should be chosen as the platform? Other chapters in this volume address the question of how a platform should be optimally chosen in depth. We will not dwell on this point, but rather adopt the traditional approach in automotive engineering, whereby the chassis (see Fig. 6) is used as the platform. There are two distinct steps in the single platform problem: (i) choosing the module which is to be used as the platform, and (ii) choosing "optimal" values for the design variables associated with the platform. Of the three design variables associated with the chassis, we let ground clearance (GC) be adjusted freely via the suspension and wheel size, while wheelbase (WB) and track (WT) are constrained as platform variables.

Table 16: Platform definition

\begin{tabular}{|c|c|c|}
\hline \multicolumn{3}{|c|}{ Platform: Chassis (module $k=2)$} \\
\hline $\mathbf{x}(\mathbf{3})$ & $\mathbf{x}(\mathbf{4})$ & $\mathbf{x}(\mathbf{5})$ \\
\hline WT & $\mathbf{W B}$ & $\mathbf{G C}$ \\
\hline$[$ in] & {$[$ in] } & {$[$ in] } \\
\hline common & common & unique \\
\hline
\end{tabular}

Case 2: We will first assume that we have only a single platform $(p=1)$ and that we will derive all 7 variants from it. This is implemented by forcing all variants to use the same settings for WT and WB, while allowing unique settings for all other design variables. Table 17 shows the settings for an initial guess of the platform, whereby the WB and WT were chosen as the (demand) weighted average of the WB and WT over all optimized variants shown in Table 15 (bottom):

$$
\begin{aligned}
& W T_{\alpha}=x(3)_{\alpha}=\frac{1}{D_{T}} \sum_{i=1}^{m} D_{i} x_{3, i}^{*} \\
& W B_{\alpha}=x(4)_{\alpha}=\frac{1}{D_{T}} \sum_{i=1}^{m} D_{i} x_{4, i}^{*}
\end{aligned}
$$


All variants built from this single $\alpha$-platform are assessed in terms of functional performance, value and demand as described in the single product model described in Section 3. It is assumed that the only effect that the platform has on the upper branch of the model framework (Fig.3) is to constrain the value of the platform variables. The lower branch is modified in terms of variable costs and platform costs according to Eq. (38b) and Eq. (43). A tradeoff therefore exists between value (and concomitant demand) losses of individual variants in the upper branch of the framework and variable and fixed cost savings in the lower branch of the framework.

Table 17 shows the design variable settings for the single platform case $(\alpha)$, where the platform variables have been chosen - somewhat naively - according to Eq. (44). The platform is highlighted in gray.

Table 17: Initial guess at a single vehicle platform $(\alpha)$

\begin{tabular}{|c|c|c|c|c|c|c|c|c|c|c|}
\hline \multicolumn{2}{|c|}{ Segment } & $\mathbf{x}(\mathbf{1})$ & $\mathbf{x}(2)$ & \multicolumn{2}{|c|}{$\alpha$-platform } & $\mathbf{x}(\mathbf{5})$ & $x(6)$ & $x(7)$ & $\mathbf{x ( 8 )}$ & $\mathbf{x}(9)$ \\
\hline & & & & & & & In & | In & {$[\mathrm{mm}]$} & [in] \\
\hline \multirow{7}{*}{ 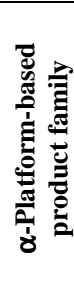 } & SML & 14.1 & 1720 & 61.8 & 111.5 & 4.9 & 53.0 & 168.1 & 186 & 14 \\
\hline & MED & 20.3 & 2421 & 61.8 & 111.5 & 4.9 & 57.9 & 200.3 & 212 & 15 \\
\hline & LRG & 19.2 & 3283 & 61.8 & 111.5 & 5.2 & 56.7 & 210.5 & 214 & 16 \\
\hline & SPT & 14.5 & 3106 & 61.8 & 111.5 & 6.3 & 49.7 & 191.5 & 200 & 15 \\
\hline & VAN & 20.0 & 3076 & 61.8 & 111.5 & 7.3 & 69.1 & 196.9 & 212 & 15 \\
\hline & SUV & 15.0 & 2679 & 61.8 & 111.5 & 7.7 & 68.2 & 173.0 & 236 & 15 \\
\hline & TRK & 24.8 & 3504 & 61.8 & 111.5 & 6.7 & 67.0 & 207.4 & 236 & 16 \\
\hline
\end{tabular}

How well would such a platform-based product family perform? We can evaluate the performance of the product family by computing the expected sales and revenue of each vehicle as before, except that we replace the "optimal" settings of variables WT and WB from Table 15 with the settings for the platform (gray shaded) shown in Table 17. We therefore expect each variant to be somewhat suboptimal compared to the optimized variants, which is expected to result in a reduction in value and sales of each variant since the price has been kept constant. On the other hand there will be a benefit through lowering the variable cost of the platform module (chassis) across the product family (Eq. 38b) and concomitant lower fixed costs (Eq. 43). Table 18 compares the performance of the optimized product family without platforming (left), with the performance of a product family where all variants use the initial $\alpha$-platform design (right) according to Eq. (44).

Table 18: Comparison of no-platforming with single vehicle platform $(\alpha)$ strategy

\begin{tabular}{|l|c|c|}
\hline & No Platforming & Single $\boldsymbol{\alpha}$-Platform \\
\hline Total NPV (11 y) & $\mathbf{\$ B ~ 7 6 4 . 8}$ & $\$ \mathrm{~B}(577.3) \mathbf{5 9 8 . 1}$ \\
\hline $\mathrm{D}_{\mathrm{fam}}$ & $\mathbf{1 3 , 2 2 9 , 9 8 2}$ & $\mathbf{1 0 , 2 0 8 , 7 5 7}$ \\
\hline
\end{tabular}

The result is that the use of the single platform shown in Table 17 leads to a $20.5 \%$ drop in NPV and a $22.8 \%$ drop in sales volume, while the estimated cost benefit of the platform is only estimated to be about $3.6 \%$. 
How can this be explained? Table 19 shows a demand and value comparison of the product families without (left) and with platforming (right). The use of the ill-conceived initial platform has caused a loss of value for most vehicles, with the exception of the SPT car. In the case of the small sedan (SML) the model predicts that there would be no demand at all for such a car in its particular market segment.

Table 19: Demand and value comparison

\begin{tabular}{|l|c|c|c|c|}
\hline & \multicolumn{2}{|c|}{ No Platforming } & \multicolumn{2}{c|}{ Single $\boldsymbol{\alpha}$-Platform (Table 17) } \\
\hline & Demand [units] & Value [\$] & Demand [units] & Value [\$] \\
\hline SML & 562,217 & 27,781 & 0 & 0 \\
\hline MED & $1,631,753$ & 49,047 & $1,355,984$ & 46,784 \\
\hline LRG & 483,815 & 80,795 & 389,732 & 76,482 \\
\hline SPT & 267,309 & 72,974 & 278,151 & 73,880 \\
\hline VAN & 397,535 & 51,707 & 189,251 & 47,148 \\
\hline SUV & $3,506,711$ & 103,215 & $2,383,818$ & 86,853 \\
\hline TRK & $6,380,642$ & 75,294 & $5,611,820$ & 70,238 \\
\hline Total & $\mathbf{1 3 , 2 2 9 , 9 8 2}$ & & $\mathbf{1 0 , 2 0 8 , 7 5 7}$ & \\
\hline
\end{tabular}

Why is this so? Plugging in the values of the design vector for the platform-based SML vehicle in the engineering performance model (Section 3.3) leads to the functional attributes shown in Table 20. The critical, baseline and ideal values for this particular market segment are also shown. We see that the platform is oversized for this small vehicle and causes one of the functional attributes, $f(3)$, to fall outside the critical range which renders the vehicle value-less according to the model. One may argue whether or not a small sedan with a towing capacity of only $\sim 475 \mathrm{lbs}$, but otherwise satisfying attributes, is indeed without value.

Table 20: Functional attributes and critical, ideal and baseline values for $\alpha$-platform based SML vehicle

\begin{tabular}{cccccc}
\hline \hline Values & $f(1) P V$ & $f(2) C V$ & $f(3) T C$ & $f(4) F E$ & $f(5) A C$ \\
\hline Type & LIB & LIB & LIB & LIB & SIB \\
Units & {$[\mathrm{cft}]$} & [cft] & {$[\mathrm{lbs}]$} & {$[\mathrm{mpg}]$} & {$[\mathrm{sec}]$} \\
Critical values $f_{C}$ & 32 & 0 & $\mathbf{8 0 0}$ & 8 & 20 \\
Ideal Values $f_{I}$ & 205 & 88 & 12,600 & 45 & 2.5 \\
Baseline Values $f_{0}$ & 88.9 & 13.2 & 1,143 & 31.4 & 9.6 \\
SML $\left(\alpha\right.$-based) $f_{i}$ & 85.9 & 24.9 & $\mathbf{4 7 4 . 8}$ & 28.0 & 10.75 \\
\hline \hline
\end{tabular}

Should we therefore conclude that platforming is not appropriate for this particular product family? Not necessarily. First, the $\alpha$-platform has not (yet) been optimized for the product family, since we have only chosen an initial guess, Eq. (44), for the platform design. Second, one would have the opportunity to adjust the non-platform design variables in response to the platform settings.

We first consider the performance of the product family over a wide range of platform designs, by varying WB and WT over the entire range of occurrences in the vehicle database (Table $\mathrm{C} 1$ ). The narrowest vehicle has a wheel track of 54.4" (TRK), the widest one has a WT of 68.4" (SUV). The shortest wheelbase is 89.2" (SPT), the longest 
one is 137.10" (SUV). We accept these as the lower and upper bounds on the dimensions that any platform can take.

Fig.20 shows the result of a comprehensive search over platform designs between the lower and upper bounds for the two platform variables (WB and WT). The plot is shown as a contour chart. The result suggest that the best possible product family NPV that can be achieved with a single platform is \$B 649.4 at platform settings of WT $\alpha *=57.9$ " and $\mathrm{WB} \alpha *=104.2$ ". The figure suggests that this "optimum" single platform design is relatively flat, but that it does not achieve as good a performance as the family of pointoptimized vehicles without platforming (\$B 764.8).

Again, we might conclude that platforming is not an appropriate strategy at this point. Note however, that the individual variants have not been re-optimized for the use of this optimized platform. At this point one may enter an optimization loop where the platform and variants are optimized in turn until convergence is achieved. Several singlelevel and bi-level approaches have been proposed to solve this classical problem (Simpson 2003).

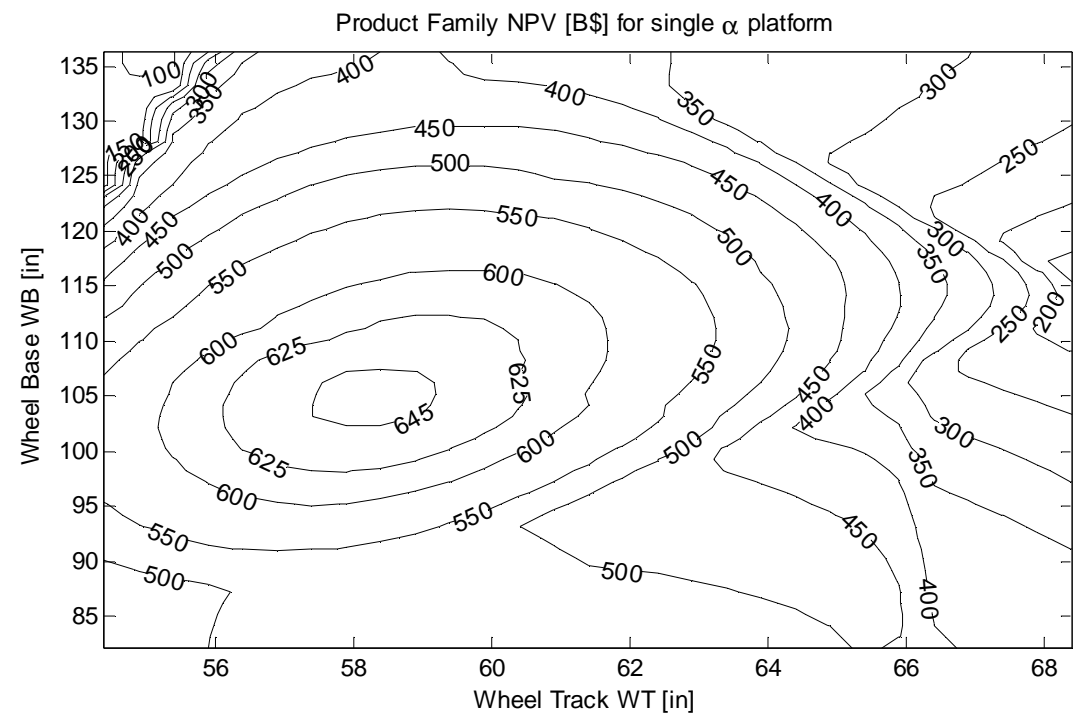

Figure 20: Product family NPV [\$B] as a function of $\alpha$-platform design (WT,WB)

Instead, we focus on the features of Fig.20 which suggest that the decision space is not smooth and that the use of multiple platforms might be more appropriate. This is motivated by the fact that the "extent" of the single $\alpha$-platform suggested so far might be too large. In fact, industrial practice confirms that no single automotive manufacturer offers a product family across the seven market segments (Fig.18) that is built from a single platform, primarily because the requirements of the different variants would be too diverse. 


\section{MULTI-PLATFORM STRATEGY}

\subsection{Case 3: Platform Extent Optimization}

In this section we consider the results when multiple platforms are allowed. In this case there could be $\alpha, \beta, \gamma, \ldots$ platforms. The questions that arise are situated one level above the single platform problem. In addition to choosing the "optimal" design variable settings for each platform, we must ask:

- What is the best number of platforms, $p$, to implement?

- What is the optimal assignment $A_{p}$ of the $m$ variants to the $p$ platforms?

We recognize that any multi-platform strategy occupies the intermediate space between two extremes: no-platforming $(p=m)$ and single-platforming $(p=1)$. A number of manufacturing firms with $m>3$ product variants are using multiple platforms to support their product families. However, oftentimes the use of multiple platforms arises historically, e.g. via acquisition of other firms rather than based on systematic considerations.

Here, we formulate the multi-platform (platform extent) problem as a weighted least squares optimization problem. This is an approximation to what could be solved as a tri-level optimization problem. In a tri-level formulation the individual variants would be optimized at the lowest level, the platforms at the intermediate level and the variantplatform assignment would be solved at the highest level. Such a multi-level scheme is likely to be intractable, or at a minimum it will converge poorly. Instead, the weighted least-squares problem is first solved for the single platform case $(p=1)$ and subsequently for each case of an additional platform $(p=p+1)$ until we reach the case where $p=m(=$ no platforming):

$$
\begin{aligned}
& \text { find } \mathbf{x}_{\text {platform }}, A_{p} \text { such that } \\
& \min \sum_{i=1}^{p} \sum_{j=1}^{m} A_{p, i, j} \cdot D_{j} P_{j}\left[\sum_{k=1}^{n_{\text {rplatform }}}\left(\mathbf{x}_{\text {plafform }, i, k}-\mathbf{x}_{j, k}{ }_{j, k}\right)^{2}\right. \\
& \forall p=\{1,2, \ldots, m\}
\end{aligned}
$$

The cost function contains the Euclidian distance between the design variable settings of the $j$-th optimized variant (without platform) $\mathbf{x}^{*}{ }_{j}$ and the platform settings $\mathbf{x}_{\text {platform, }}$ of the $i$-th platform to which the $j$-th product variant has been assigned. The assignment is done via a binary assignment matrix $A_{p}$, which has $p$ rows and $m$ columns. For example, if variant $j=2$ is assigned to platform $i=3$, then the entry $A_{p}(3,2)=1$. Assuming that a product variant can only be built from a single platform, all other entries in the $2^{\text {nd }}$ column of $A_{p}$ will be zero. The Euclidian distance between "ideal" variant 
settings and the platform settings are weighted by the expected revenue (demand times price) of the variant. ${ }^{\dagger+\dagger \dagger}$

The result of this optimization is, for each number of allowed platforms, an "optimal" design of each platform as well as the corresponding platform-to-variant assignment, $A_{p}$. We will demonstrate this procedure for the automotive platforming problem developed in Section 4.

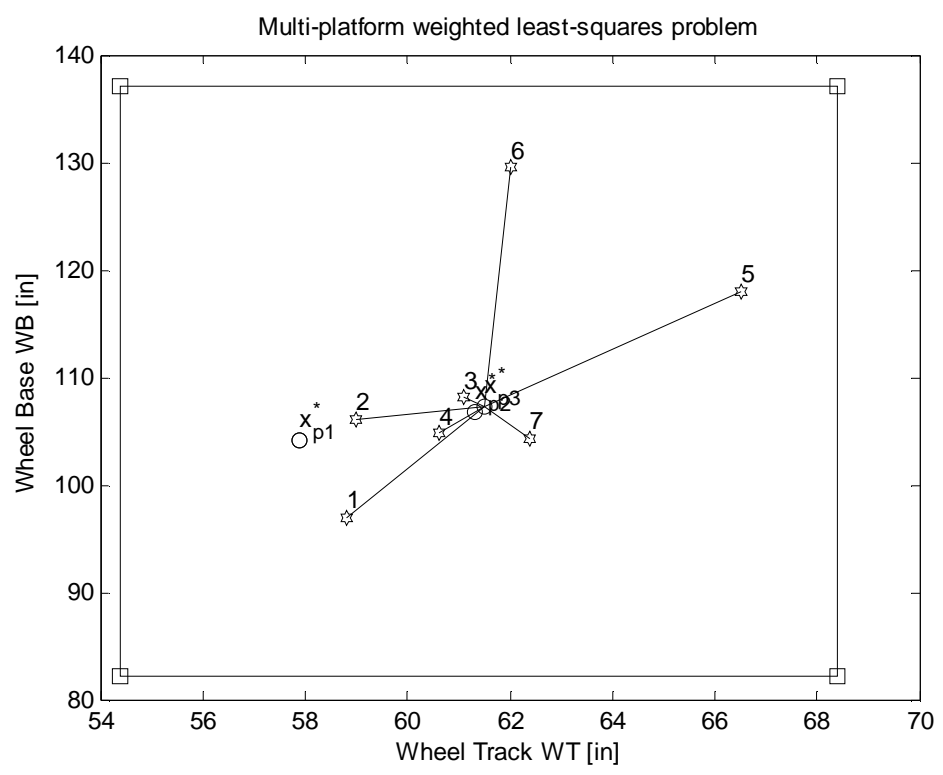

Figure 21: Weighted least-squares optimization of product platform $(p=1)$

Figure 21 shows the result of optimizing a single product platform $(\alpha)$ with the weighted least squares objective (Eq. 45). The hexagons labeled 1-7 in Fig.21 indicate the position of the ideal settings for the variants (SML through TRK) from Table 15 (bottom). Three different potential single platforms are shown:

- $\quad x_{p 1}^{*}$ : optimal single platform from NPV exhaustive search, Fig.20 (NPV= \$B 649.4)

- $\quad x_{p 2}^{*}$ : optimal single platform with Simulated Annealing, Eq. (45) (NPV= \$B 605.1)

- $\quad x_{p 3}^{*}$ : optimal single platform with gradient search, Eq. (45) (NPV=\$B 603.2)

In the figure the variant-to-platform assignment is shown by connecting the variants to their respective platform $\left(\mathbf{x}_{\mathrm{p} 3}\right.$ in this case). We note that using the approximate least squares objective metric, Eq. (45), causes a $7.1 \%$ penalty compared to using the more

$+1+t \dagger$ Note that the demand and revenue of the $j$-th variant in Eq.(45) is estimated based on the baseline (case 1) without platforming. 
expensive NPV metric directly. We will accept this penalty in order to solve the multiplatform problem.

Fig. 22 shows a block diagram of the multi-platform (platform extent) solution method. First, it is recommended to solve the two bounding cases $(p=1, p=m)$. This gives an initial guess for the product platform designs for the intermediate cases where $(1<p<m)$. For a given number of platforms, the platform designs are placed in the design space (see Fig. 21) and are perturbed, e.g. with Simulated Annealing (SA) or another search algorithm. Next, the optimal assignment $A_{p}$ from variants-to-platforms is found that will minimize the weighted objective function (Eq.45). This loop is repeated until convergence is achieved and the optimal platform designs $\mathbf{x}_{\mathrm{p}^{*}}$ and assignment $A_{p}$ is obtained for $p=\{2,3 \ldots m-1\}$.

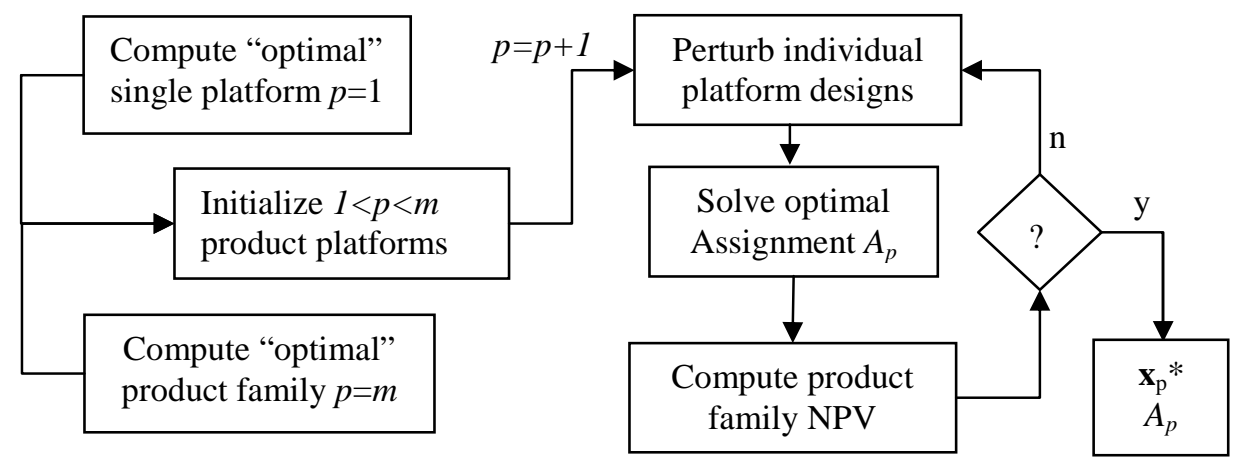

Figure 22: Multi-platform optimization procedure

Figure 23 shows the result of solving the multi-platform problem with the above method. The predicted product family NPV is shown as a function of the number of allowed product platforms. On the left side $(p=1)$ we find the case of a single platform from Section 4.3, on the right we find the no-platforming case from Section 4.2. The latter case is identical with the multi-platform case for $m=p=7$, as each platform, automatically converges to the optimal settings for each variant. In that case each "platform" only supports a single variant.

The bar chart (Fig.23) shows the average results for five runs of the simulated annealing algorithm. We can clearly see that the single-platform case shown in Fig.21 is the worst strategy. The results are relatively flat for $2<p<6$, primarily because the WB and WT requirements for variants 3,4 and 7 are relatively similar, as can be seen in Fig.21. The best strategy appears to be case where 6 platforms are used. In that case only the MED and LRG sedans share a common platform. The strategy with $\mathrm{p}=5$ platforms is nearly equivalent. 


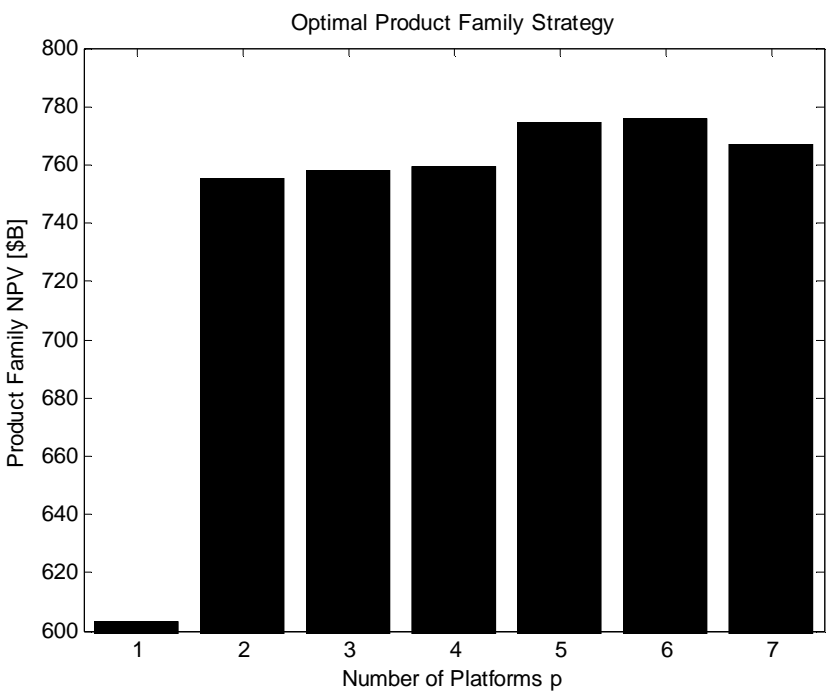

Figure 23: NPV as a function of the number of platforms

Perhaps more interesting than the raw NPV predictions for each platform strategy (which are subject to model uncertainty), is the suggested variant-to-platform assignment. For the cases shown in Fig. 23 we can find the best assignment of variants to platforms (Table 21):

Table 21: Optimal variant-to-platform assignment

\begin{tabular}{|c|c|c|c|c|c|c|c|}
\hline$A_{p}$ & 1-SML & 2-MED & 3-LRG & 4-SPT & 5-VAN & 6-SUV & 7-TRK \\
\hline$p=1$ & 1 & 1 & 1 & 1 & 1 & 1 & 1 \\
\hline$p=2$ & 2 & 2 & 2 & 2 & 1 & 1 & 2 \\
\hline$p=3$ & 2 & 3 & 3 & 2 & 1 & 1 & 2 \\
\hline$p=4$ & 3 & 4 & 4 & 3 & 1 & 2 & 3 \\
\hline$p=5$ & 5 & 3 & 3 & 1 & 4 & 2 & 1 \\
\hline$p=6$ & 2 & 6 & 6 & 3 & 4 & 1 & 5 \\
\hline$p=7$ & 5 & 7 & 1 & 3 & 6 & 4 & 2 \\
\hline
\end{tabular}

In the single platform case $(p=1)$ all variants are forced to use the same platform (1) and there is no choice, except for the choice of design variables of that single platform. When a second platform is allowed $(p=2)$, the VAN and SUV are assigned a new platform that is optimized for a larger wheel track and wheel base. This is intuitive as these two particular vehicles (5-VAN, 6-SUV) can also be seen as the outliers in Fig. 21 . With $p=3$, the MED and LRG sedans share a platform, while the SML, SPT and TRK products share another platform. With $p=7$, every variant is customized and has its own "platform". A deeper understanding of variant-to-platform assignment can be gained by plotting the position of the $p$ platforms and assignment of variants in the same graph (Fig. 24). 


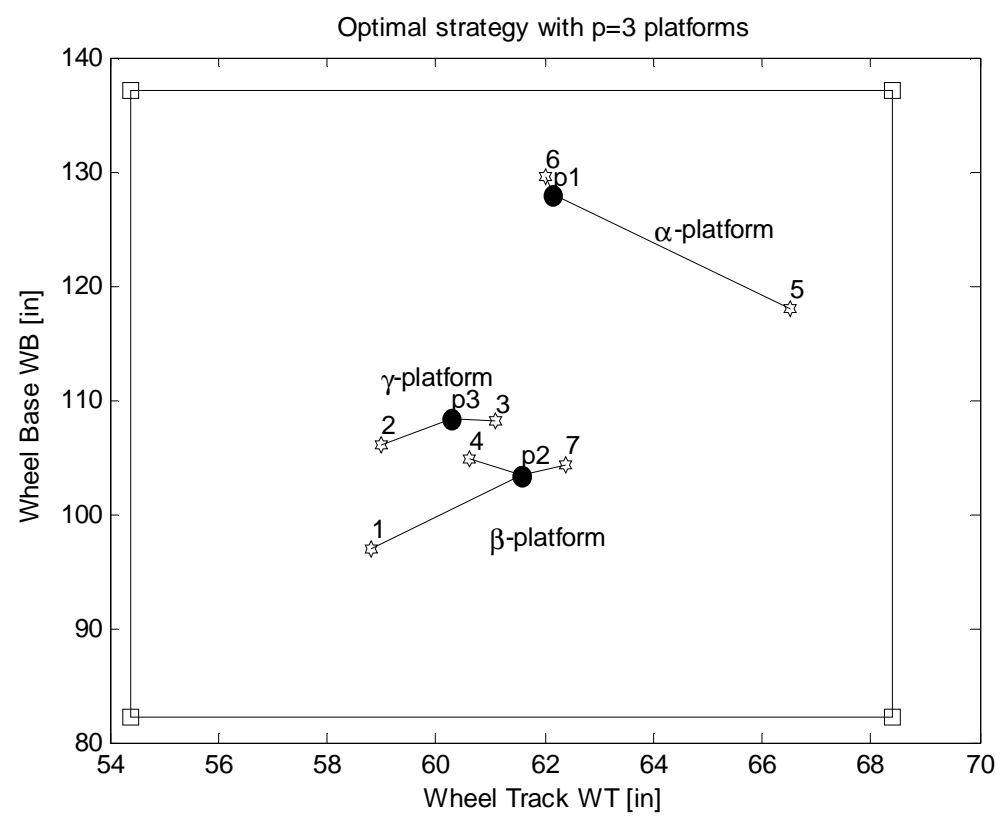

Figure 24: Optimal strategy with $p=3$ platforms

Figure 24 shows that in the case of $p=3$, the $\alpha$-platform would support the VAN (5) and SUV (6), but would be heavily weighted towards the SUV based on its more significant generated revenue, $P_{6} D_{6}$. The $\beta$-platform would support the SML (1), SPT (4) and TRK (7) vehicles and be weighted towards the TRK, since this represents one of the largest market segments (in the North American database). Finally, the $\gamma$-platform would support the MED (2) and LRG (3) sedans with a longer wheelbase, but slightly narrower wheel track than the $\beta$-platform.

\subsection{Sensitivity Analysis}

From a decision theory standpoint one would like to know how robust the answers in Fig. 23 are to various assumptions made throughout the modeling framework outlined in Section 3 and shown in Fig. 3. We first list what are believed to be the key assumptions that are likely to affect the optimal multi-platform strategy (Table 22). We then modify one of those assumptions to gauge its effect on the NPV results.

Table 22: Assumed parameters with potential impact on optimal platform strategy

\begin{tabular}{|l|l|l|}
\hline Parameter & Eq. & Potential effect on platforming \\
\hline$a_{o}-a_{6}$ & $(6)-(13)$ & $\begin{array}{l}\text { RSM for engineering performance can be of limited validity } \\
\text { if a variant is built on a platform that is far from the average } \\
\text { of the data that was used to construct the original RSM. }\end{array}$ \\
\hline$f_{C}, f_{I}$ & $(15)$ & $\begin{array}{l}\text { Critical and ideal values for the product value model can } \\
\text { impact the valuation of a platform-derived product variant } \\
\text { by more or less penalizing deviation from the optimal }\end{array}$ \\
\hline
\end{tabular}




\begin{tabular}{|c|c|c|}
\hline & & design of non-platformed variants. \\
\hline$N$ & (17) & $\begin{array}{l}\text { The number of competitors considered in the demand } \\
\text { prediction model will affect the degree to which } \\
\text { platforming can lead to a market share penalty or benefit. }\end{array}$ \\
\hline$E_{2}$ & (20) & Price elasticity affects demand via $K(V-P)$ \\
\hline$\alpha_{i}$ & $(28),(43)$ & $\begin{array}{l}\text { The amount of operating profit (before platforming) will } \\
\text { influence how helpful platforming is for improving NPV } \\
\text { Platforming is much more interesting when } \alpha_{i} \text { is low, } \\
\text { because manufacturing cost savings through platforming } \\
\text { can make a more substantial contribution to profit. }\end{array}$ \\
\hline$\phi_{i}$ & $(29),(43)$ & The operating leverage affects the value of platforming. \\
\hline$S, B$ & $(32),(33)$ & $\begin{array}{l}\text { A strong manufacturing learning curve }(S \text { small }) \text { is expected } \\
\text { to strongly favor platforming, because the benefits of reuse } \\
\text { are captured in the variable manufacturing costs. }\end{array}$ \\
\hline$\beta_{i k}$ & $(35)$ & $\begin{array}{l}\text { The } \% \text { content that the platform constitutes relative to the } \\
\text { whole product will impact the estimated benefits of } \\
\text { platforming. We assumed } 0.3<\beta_{i 2}<0.4 \text { for the chassis, but } \\
\text { if this is substantially smaller, then the choice of the chassis } \\
\text { as the platform would be less beneficial. }\end{array}$ \\
\hline$M_{R D}, M_{P}$ & $(40)$ & $\begin{array}{l}\text { The R\&D budget and budget for facility and tooling upkeep } \\
\text { and maintenance will impact platform benefits. Platforming } \\
\text { can also impact what those budgets need to be. }\end{array}$ \\
\hline$r$ & $(41)$ & $\begin{array}{l}\text { The discount rate will affect the benefit of platforming in } \\
\text { the future. A platform is an investment in future production } \\
\text { capability. So if } r \text { is very large }(>10 \%) \text {, future benefits of } \\
\text { platforming will be washed out of the NPV calculations. }\end{array}$ \\
\hline$\overline{D_{\text {plant }}}$ & $(43)$ & $\begin{array}{l}\text { Platforming is beneficial in industries where plant capacities } \\
\text { have to be large (e.g. } D_{\text {plant }}>100,000 \text { ) due to economies of } \\
\text { scale and where multiple variants can be built in the same } \\
\text { plant. A larger } D_{\text {plant }} \text { will benefit platforming. }\end{array}$ \\
\hline
\end{tabular}

We will investigate the effect that the learning curve factor, $S$, has on the optimal platform strategy. So far, we have assumed that $S=0.95$ across all fabrication and assembly processes, see Eq.(33). This is a relatively conservative value, as it assumes that doubling the production quantity will allow to lower the per-unit cost to $95 \%$ of its previous value. We therefore set $\mathrm{S}=0.80$ and repeat the multi-platform optimization process shown in Fig. 22.

The results with $S=0.80$ are shown in the bar chart of Fig.25. We can see that the magnitude of the predicted NPV's is significantly higher than in Fig.23. This is not due to increases in sales volumes, but rather due to substantial savings in variable manufacturing costs across the product family, $C_{\text {fam }}$, Eq. (38b). The second effect of interest is that the no-platforming strategy $(p=7)$ is now clearly inferior and one should focus on strategies where $3<p<6$. The actual choice of strategy in practice will also depend on factors that are not represented in the model. This includes dealing with legacy platforms and product variants which cannot be replaced quickly, mainly due to large capital investments (e.g. tooling) that have to be amortized. 


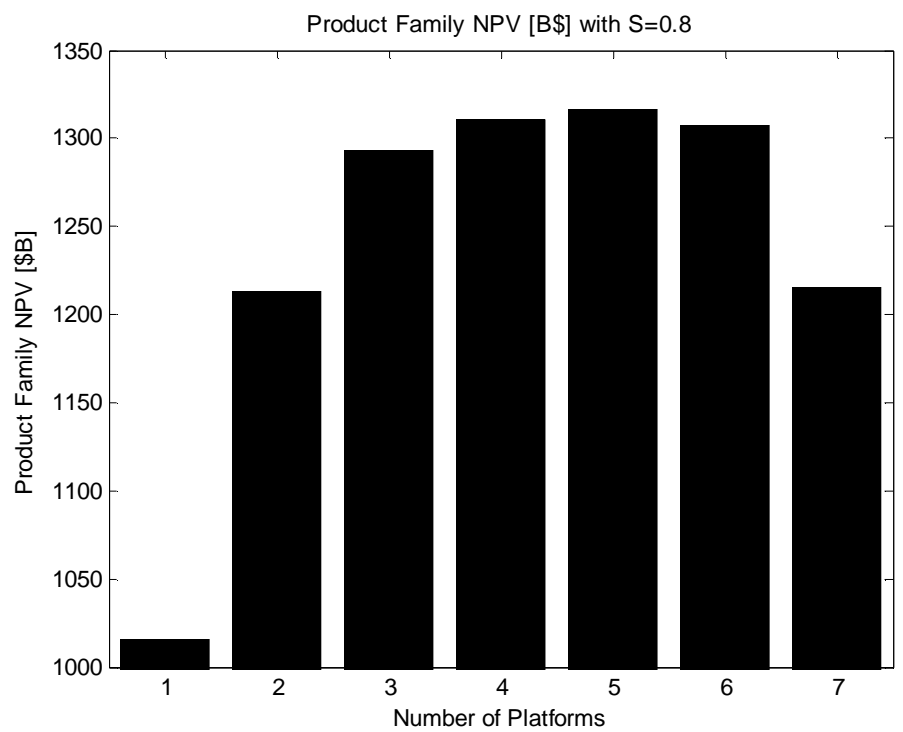

Figure 25: NPV as a function of the number of platforms, $S=0.8$

At the beginning of the chapter we had suggested that there are multiple ways of viewing and interpreting the concept of "platform extent". We mentioned four views:

1. design space view: range of design variables $\mathbf{x}$ covered by platform, see Fig. 24

2. market segmentation view: see Fig.26a for $\beta$-platform from Fig.24

3. value-price view: $V-P$ space spanned by platform, see Fig.26b for $\beta$-platform

4. functional view: radar plot of variant functional attributes, see Fig.26c

Fig.26 shows the platform extent of the $\beta$-platform from Fig.24. This platform was suggested by the optimized multi-platform strategy where $p=3$ platforms were utilized. Rather than drawing notional pictures (as in Fig. 2), platform extent can now be crisply defined, computed and visualized.

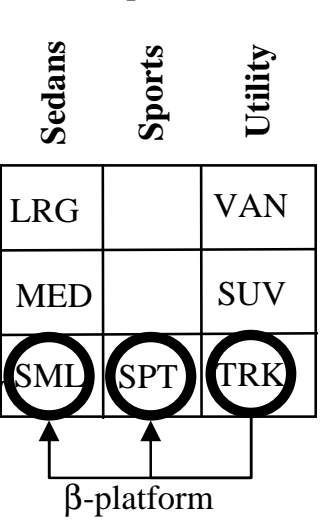

(a) Market Segmentation view

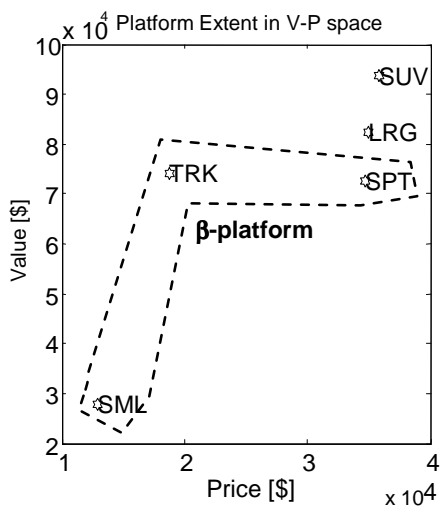

(b) P-V view

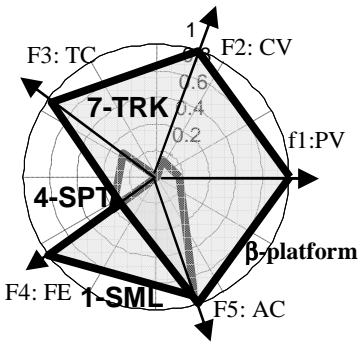

(c) Functional view

Fig.26 Three different views of product platform extent 


\section{DISCUSSION AND SUMMARY}

\subsection{Discussion}

While the product framework (Section 3), single platform case (Section 4) and multiplatform method (Section 5) presented an end-to-end view, it must be acknowledged that a significant number of simplifying assumptions were made. In some cases such assumptions are necessary and without significant consequences on the final answer. In other circumstances one must be more cautious when interpreting the results of such analysis. What follows is a list of aspects that need to be considered beyond what was discussed in this chapter:

- Pricing Strategy: Demand (and revenue) are significantly impacted by setting transaction prices for individual product variants. Instead of leaving prices at their "optimal" values as was done here, they are often adjusted based on seasonal factors, competitive action or inventory levels.

- Iteration Loops: Setting platform variables and variant variables is often done in an iterative fashion. So, the optimal variants shown in Table 15 could be reoptimized after finding the optimal single or multi-platform strategy. This variant re-optimization could in turn trigger a revision of the platform strategy.

- Electronics \& Software: The software and electronics content of many products (including automobiles) is constantly increasing. The product modeling framework (Fig. 3), however, is mainly geared towards electro-mechanical systems. Multi-platforming for products with significant software and electronics content is in need of future research.

- R\&D-centric businesses: The benefits of platforming in this chapter were mainly captured via reduction of variable (Eq. 38b) and fixed manufacturing costs (Eq.43). In a number of businesses, however, the main reason for platforming is related to saving time and cost in research and development. It is still unclear how multi-platform strategies could be evaluated in that context.

- BOF vs. BFI Architecture: Section 3.2 on product architecture alluded to the fact that cars and trucks are typically built from either a body-frame-integral or body-on-frame architecture. It is difficult, if not impossible, to merge vehicles of such different architectures onto the same platform, even if their "raw" design variable settings might suggest to do so.

- Product detail: As mentioned previously it should be possible to solve the platform extent problem directly (i.e. down to individual parts and details) for simple products with up to $7^{3} \sim 300$ components (coffee makers, simple cameras ...). For systems that are more complex (cars, airplanes, complex electronics) it is likely that one has to resort to an abstracted, higher level representation of the product.

- Technical model fidelity: It was demonstrated that the RSM engineering performance model used her was accurate within 6-11\%, on average. For some industries the use of such simplified models might be acceptable, for others it might be misleading. 
- Soft attributes: The model suggested that a firm should strive to set all "soft" attributes to the maximum value, e.g. a J.D. Power rating of 5 in all categories. In reality this will be difficult to achieve and improvement of soft attributes will require resources. This tradeoff has not been modeled, but it has been suggested that one of the true benefits of platforming (and multi-platforming) is that a firm can dedicate more attention and resources to the soft attributes (e.g. styling, interiors) because the main functionality of the product variants has already been "built-in" to the one or more platforms.

- Functional attributes: One needs to be careful to interpret and monitor the evolution of the functional value weighting factors, $\gamma$. First, these can (and do) evolve over time and new functional attributes can emerge that were not considered when the platform(s) were originally conceived.

- Flexible product platforms: All platforms considered in this chapter were considered as fixed, i.e. once WT and WB were chosen they could not be changed. An important research topic is the embedding of flexibility in product platforms. For example, we see in Fig. 24 that the $\beta$ and $\gamma$-platforms are not too different in terms of $\Delta \mathrm{WT}(\sim 2 ")$ and $\Delta \mathrm{WB}(\sim 8 ")$. One could conceivably agree on a compromise width (WT) and design the platform flexibly such that it could be stretched lengthwise (WB), say from 100-110" without significant switch costs. Such flexibility might require upfront investment, but might allow reducing the "optimal" number of platforms suggested under the assumption that each platform is fixed.

\subsection{Summary}

In this chapter we have discussed the relevance and challenges of the multi-platform problem that all manufacturing firms face who offer many product variants $(m>3)$. The problem is challenging and multi-faceted and requires the adoption of an end-to-end modeling framework (Fig.3) that connects six different domains: product architecture, performance engineering, value modeling, market demand modeling, manufacturing costing and investment finance. Various methods and techniques exist to populate these sub-domains, but we believe that the interface quantities (design variables $\mathbf{x}$, functional attributes $\mathbf{f}$, value $V$, cost $C$, demand $D$ and $N P V$ ) are invariant.

The first step in answering the platform extent problem (how many platforms?, what is the optimal variant-to-platform assignment?) is to solve the two extreme cases: $p=m$ (no platforming) and $p=1$ (single platform). These cases can be solved with a number of single and bi-level optimization schemes that have been proposed in the literature. To solve the multiplatform problem $(1<p<m)$ we suggest a weighted least squares formulation (Eq. 45) rather than setting up an intractable tri-level formulation. In the least squares problem, we allow $p$ platforms and attempt to position these in the design space such that the $m$ variants can be optimally assigned to minimize the objective function. The objective function is a compound Euclidian distance metric, whereby the distance of each variant to its assigned platform is weighted by its projected revenue per unit time.

The framework has been demonstrated for a (hypothetical) product family of 7 automotive model variants. The results indicate that a 5-platform strategy appears most suitable. The model suggests that forcing these diverse products onto a single platform is 
counterproductive, because such a platform extent would be too large, leading to significant value losses of the associated variants in their particular market segments. Industrial practice mirrors this result as automotive platforms are typically leveraged only within larger market segments (e.g. mid-size sedans), but rarely between segments.

The framework presented here is believed to be generally applicable, subject to the caveats mentioned in the previous section. Fig.27 shows that the "Platform Family Plan" occupies a central place in the corporate strategy of the for-profit manufacturing firm.

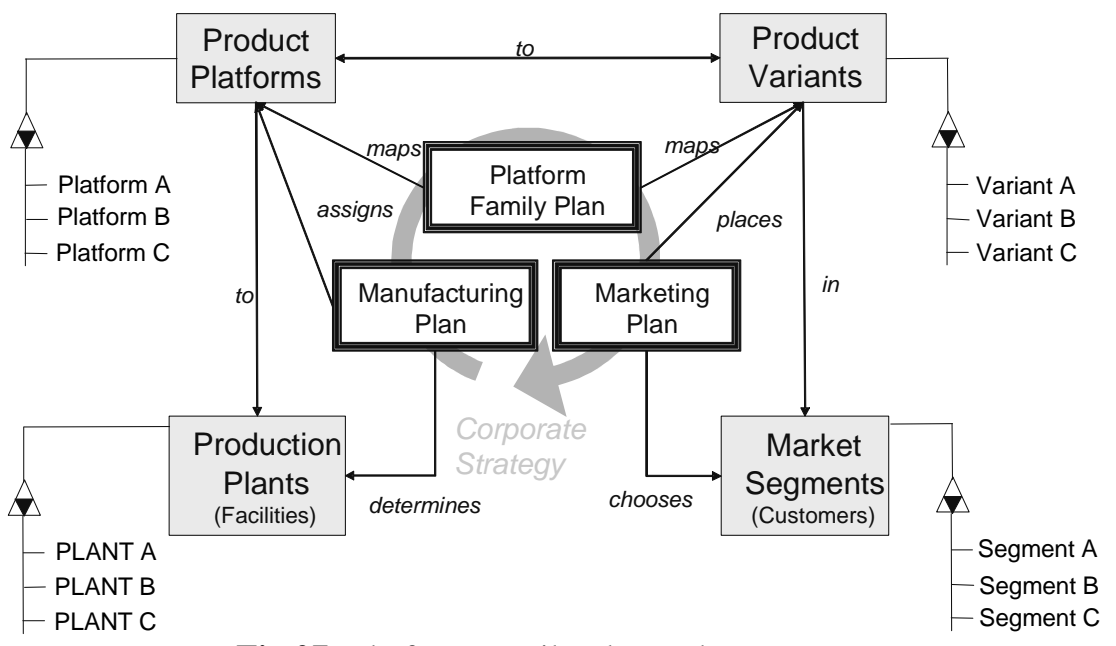

Fig.27: Platform Family Plan and corporate strategy

The Marketing Plan assigns product variants to market segments and decides which of the firm's products will compete against which of the competitor's products. The platform extent problem therefore requires that both the market segments and the types and number $(m)$ of desired product variants are known. The Platform Family Plan assigns variants to platforms and embodies key engineering decisions about architecture and design of the platforms and variants. Finally, the manufacturing plan assigns platforms to manufacturing plants. This is important as plants are typically equipped with machines and tooling that are geared towards a limited set (mostly one) platform. One of the most important complications in the manufacturing plan is that plants should be made to run at or near capacity. One of the main reasons for success (or failure) in achieving profitability in the automotive industry relates to whether or not plant capacity can be utilized at a high rate (say >80\%). This depends not only on the market success of individual models, but also on the flexibility to balance loads of a multi-platform product family across multiple manufacturing facilities. 
NOMENCLATURE

\begin{tabular}{|c|c|}
\hline $\mathrm{AC}$ & acceleration time from $0-100 \mathrm{~km} / \mathrm{h}$, [sec] \\
\hline $\mathrm{CO}$ & comfort, $[1-5]$ \\
\hline $\mathrm{CV}$ & cargo volume, $[\mathrm{cft}]$ \\
\hline $\mathrm{CW}$ & curb weight, [lbs] \\
\hline ED & engine displacement, $[\mathrm{ccm}]$ \\
\hline FC & fuel tank capacity, [gallons] \\
\hline FE & combined fuel economy, $[\mathrm{mpg}]$ \\
\hline GC & ground clearance, [in] \\
\hline HP & horsepower rating, [hp] \\
\hline HT & total height, [in] \\
\hline LT & total length, [in] \\
\hline MD & mechanical dependability, [1-5] \\
\hline MQ & mechanical quality, [1-5] \\
\hline PR & price, [2002 U.S. \$] \\
\hline PV & passenger volume, $[\mathrm{cft}]$ \\
\hline SE & service experience, $[1-5]$ \\
\hline ST & styling, [1-5] \\
\hline SV & sales volume, [\# units/year] \\
\hline TC & towing capacity, [lbs] \\
\hline TD & tire diameter, [in] \\
\hline TW & tire width, $[\mathrm{mm}]$ \\
\hline WB & wheel base, [in] \\
\hline WT & front wheel track (width), [in] \\
\hline$A$ & profit, \$ \\
\hline$B$ & learning curve exponent \\
\hline$c_{i, t f u}$ & theoretical first unit costs (variable) for the $\mathrm{i}$-th product \\
\hline$c_{i, f u}^{\prime}$ & scaled theoretical first unit costs (variable) for the $\mathrm{i}$-th product \\
\hline$c_{i, f f u, k}$ & theoretical first unit costs (variable) for the k-th module of i-th product \\
\hline$C_{i}$ & variable cost of the i-th product, $\$$ \\
\hline$C_{i}^{\prime}{ }_{i}(-2)$ & variable cost of the $i$-th product with scaling and learning curve, $\$$ \\
\hline$D_{a}$ & actual sales volume (=demand), \# units per unit time \\
\hline$D_{i}$ & predicted demand for the $\mathrm{i}$-th product, \# units per year \\
\hline$f_{i}$ & i-th functional performance attribute \\
\hline f & vector of functional performance attributes \\
\hline$F_{i}$ & fixed cost for i-th product, $\$$ \\
\hline$m$ & number of variants, number of functional attributes \\
\hline$m_{P}$ & Plant and tooling investment costs a percent of sales, $\%$ \\
\hline$m_{R D}$ & Research and development costs as a percent of sales, $\%$ \\
\hline$M_{P, i, j}$ & Plant investment costs for the $\mathrm{i}$-th product in the $\mathrm{j}$-th year, $\$$ \\
\hline$M_{R D, i, j}$ & Research and development costs for the $\mathrm{i}$-th product in the $\mathrm{j}$-th year, $\$$ \\
\hline$N$ & number of competitors in a market segment \\
\hline$N_{P}$ & number of time periods of production \\
\hline$N_{R D}$ & number of time periods for research and development \\
\hline$n$ & number of design variables \\
\hline$n_{\text {mod }}$ & number of modules in product \\
\hline
\end{tabular}




$\begin{array}{ll}N P V & \text { net present value, } \$ \\ p & \text { number of platforms } \\ \mathrm{p} & \text { vector of fixed design parameters } \\ P & \text { product price, } \$ \\ \mathrm{r} & \text { discount rate, typically } 0-20 \% \\ S_{i} & \text { i-th soft attribute, } 1-5 \text { (J.D. Power Rating) } \\ \mathbf{S} & \text { vector of soft attributes } \\ S & \text { learning curve factor, typically } 0.8-1.0 \\ x_{i} & \text { i-th design variable } \\ \mathbf{x} & \text { vector of design variables } \\ \mathbf{y} & \text { vector of dependant variables } \\ V & \text { product value, } \$ \\ \alpha & \text { operating margin, } 0 \text {-1 } \\ \beta_{i, k} & \text { variable cost breakdown coefficient for i-th product and k-th module } \\ \phi & \text { operating leverage (ratio of fixed to variable costs) } \\ \gamma_{i} & \text { weighting factor for i-th product attribute } \\ \gamma & \text { vector of attribute weighting factors } \\ \Pi_{i} & \text { net present value of i-th product, } \$\end{array}$




\section{REFERENCES}

Bremmer, R., "Cutting-Edge Platforms," Financial Times Automotive World, September, 1999, pp. 30-38

Caudill, M., Neural Networks Primer, San Francisco, CA: Miller Freeman Publications, 1989.

Cook, H. E., "Product Management: Value, Quality, Cost, Price, Profit and Organization", Chapman \& Hall, $1^{\text {st }}$ edition, 1997

Crawley E., "Systems Architecture", Course Notes ESD.34/16.882, Massachusetts Institute of Technology, 2001

Dori, D., Object-Process Methodology: A Holistic Systems Paradigm, SpringerVerlag Berlin Heidelberg, 2002.

Harris, D., Automotive News, March 2002

J.D. Power \& Associates, J.D. Power Consumer Center, Automotive Ratings, http://www.jdpower.com/cc/auto/index.jsp , accessed December 2004

Maier M. W., Rechtin E., The Art of Systems Architecting, CRC Press, Boca Raton, FL, 2000

Mathworks, The, "Matlab V7.0.1 (R14), Neural Network Toolbox", Natick, MA, http://www.mathworks.com, Sept. 13, 2004

Miller, G.A., "The Magical Number Seven, Plus or Minus Two: Some Limits on Our Capacity for Processing Information”, The Psychological Review, 1956, vol. 63, pp. 8197

PSA Peugeot Citroen, Annual Report 2004, released February 2005

Seepersad, C. C., Hernandez G., Allen J. K., ,A Quantitative Approach to Determining Product Platform Extent“", DETC 2000/DAC-14288, Proceedings of DETC00/DAC, 2000 ASME Design Engineering Technical Conference, September 1013, Baltimore, Maryland, 2000

Simmons, G., "U.S. Auto Market Splinters", The Detroit News, January 31, 2005

Simpson, T. W.,'Product Platform Design and Optimization: Status and Promise”, DETC2003/DAC-48717, Proceedings of DETC'03, ASME 2003 Design and Engineering Technical Conferences and Computers and Information in Engineering Conference, Chicago, Illinois, September 2-6, 2003

Srivastava A., Hacker K., Lewis K., Simpson T.W., “A method for using legacy data for metamodel-based design of large-scale systems", Structural and Multidisciplinary Optimization, 2004, 28, pp. 146-155

Ulrich, Karl T. and Steven D. Eppinger, Product Design and Development, IrwinMcGraw-Hill, New York, 3rd Edition, 2004.

Whitney, D., Mechanical Assemblies: Their Design, Manufacture, and Role in Product Development, Oxford University Press, 2004

Wong J.Y., Theory of Ground Vehicles, third edition, 2001 


\section{APPENDIX A}

Automotive database (Autopro 20002) for the United States of America.

Market Segment: medium cars (MED). Database sorted in order of decreasing sales.

Table A1: Design variables $\mathrm{x}(1)-\mathrm{x}(9)$ and dependent variables $\mathrm{y}(1)-\mathrm{y}(2)$

\begin{tabular}{|c|c|c|c|c|c|c|c|c|c|c|c|c|}
\hline \multirow[t]{3}{*}{ Brand } & \multirow[t]{3}{*}{ Model } & $\mathbf{x}(\mathbf{1})$ & $\mathbf{x}(2)$ & $\mathbf{x}(\mathbf{3})$ & $\mathbf{x}(\mathbf{4})$ & $\mathbf{x}(\mathbf{5})$ & $\mathbf{x}(\mathbf{6})$ & $\mathbf{x}(7)$ & $\mathbf{x}(\mathbf{8})$ & $\mathbf{x}(\mathbf{9})$ & $\mathbf{y}(\mathbf{1})$ & $\mathbf{y}(2)$ \\
\hline & & FC & ED & WT" & WB & GC & HT & $\mathbf{L T}$ & TW & TD & HP & $\mathrm{CW}^{\wedge}$ \\
\hline & & {$[\mathrm{g}]$} & [ccm] & [in] & [in] & [in] & [in] & [in] & {$[\mathrm{mm}]$} & [in] & [hp] & [lbs] \\
\hline Honda & Accord & 17.1 & 2254 & 61.2 & 105.1 & 3.9 & 54.9 & 186.8 & 195 & 15 & 150 & 3036 \\
\hline Toyota & Camry & 18.5 & 2362 & 60.8 & 107.1 & 5.4 & 57.9 & 189.2 & 205 & 15 & 157 & 3142 \\
\hline Ford & Taurus & 18 & 2982 & 61.6 & 108.5 & 5.4 & 56.1 & 197.6 & 215 & 16 & 155 & 3336 \\
\hline Chevrolet & Impala & 17 & 3350 & 62 & 110.5 & NL & 57.3 & 200 & 225 & 16 & 180 & 3389 \\
\hline Pontiac & Grand AM & 14.3 & 2189 & 59 & 107 & NL & 55.1 & 186.3 & 215 & 15 & 140 & 3118 \\
\hline Chevrolet & Malibu & 14.3 & 3136 & 59 & 107 & 5.5 & 56.4 & 190.4 & 215 & 15 & 170 & 3053 \\
\hline Nissan & Altima & 20 & 2500 & 61 & 110.2 & 4.1 & 57.9 & 191.5 & 205 & 16 & 175 & 3048 \\
\hline VW & Jetta & 14.5 & 1984 & 59.6 & 98.9 & 4.1 & 56.7 & 172.3 & 195 & 15 & 115 & 2945 \\
\hline Buick & Century & 17.5 & 3130 & 62 & 109 & 5.7 & 56.6 & 194.6 & 205 & 15 & 175 & 3368 \\
\hline Pontiac & Grand Prix & 17.5 & 3136 & 61.5 & 110.5 & 5.5 & 54.7 & 196.5 & 205 & 15 & 175 & 3384 \\
\hline Chrysler & Sebring & 16.3 & 2351 & 59.4 & 103.7 & 6.2 & 53.7 & 190.2 & 205 & 16 & 142 & 3099 \\
\hline Dodge & Stratus & 16.3 & 2351 & 59.4 & 103.7 & 6.2 & 53.7 & 190.9 & 205 & 16 & 147 & 3115 \\
\hline Oldsmobile & Alero & 14.1 & 2196 & 59.1 & 107 & 5 & 54.5 & 186.7 & 215 & 15 & 140 & 3010 \\
\hline Dodge & Interpid & 17 & 2736 & 61.9 & 113 & 5.1 & 55.9 & 203.7 & 225 & 16 & 200 & 3469 \\
\hline Mercury & Sable & 18 & 2982 & 61.6 & 108.5 & $\mathrm{NL}$ & 55.5 & 199.8 & 215 & 16 & 157 & 3379 \\
\hline Nissan & Maxima & 18.5 & 3500 & 60.2 & 108.3 & 5.5 & 56.3 & 191.5 & 215 & 16 & 255 & 3218 \\
\hline Saturn & L-100 & 15.7 & 2198 & 59.8 & 106.5 & 6.3 & 56.4 & 190.4 & 195 & 15 & 135 & 2989 \\
\hline Subaru & Legacy & 16.9 & 2457 & 57.5 & 104.3 & 6.1 & 55.7 & 184.4 & 205 & 15 & 165 & 3320 \\
\hline VW & Passat & 16.4 & 1781 & 59.6 & 106.4 & 4.9 & 57.6 & 185.2 & 195 & 15 & 170 & 3322 \\
\hline Mitsubishi & Galant & 16.3 & 2350 & 59.4 & 103.7 & 6.2 & 55.7 & 187.8 & 195 & 15 & 140 & 3031 \\
\hline Toyota & Avalon & 18.5 & 2995 & 61 & 107.1 & 5.1 & 57.7 & 191.9 & 205 & 15 & 210 & 3417 \\
\hline Chevrolet & MonteCarlo & 17 & 3350 & 62 & 110.5 & 5.9 & 55.2 & 197.9 & 225 & 16 & 180 & 3340 \\
\hline Hyundai & Sonata & 17.2 & 2351 & 60.6 & 106.3 & NL & 56 & 186.9 & 205 & 15 & 149 & 3217 \\
\hline Mazda & 626 & 16.9 & 1991 & 59.1 & 105.1 & 5.2 & 55.1 & 187.4 & 205 & 15 & 125 & 2961 \\
\hline Buick & Regal & 17.5 & 3790 & 62 & 109 & 5.7 & 56.6 & 196.2 & 215 & 15 & 200 & 3438 \\
\hline Oldsmobile & Intrigue & 17 & 3472 & 62.1 & 109 & 5.8 & 56.6 & 195.9 & 225 & 16 & 215 & 3434 \\
\hline Chrysler & Concorde & 17 & 2736 & 61.9 & 113 & 5.1 & 55.9 & 207.7 & 225 & 16 & 200 & 3479 \\
\hline Kia & Optima & 17.2 & 2351 & 60.6 & 106.3 & 6.1 & 55.5 & 186.2 & 205 & 15 & 149 & 3190 \\
\hline Daewoo & Leganza & 15.8 & 2198 & 59.6 & 105.1 & NL & 56.6 & 183.9 & 205 & 15 & 131 & 3157 \\
\hline Hyundai & XG350 & 18.5 & 3467 & 60.6 & 108.3 & 6.3 & 55.9 & 191.5 & 205 & 16 & 194 & 3651 \\
\hline Mitsubishi & Diamante & 19 & 3497 & 60.8 & 107.1 & 4.6 & 53.9 & 194.1 & 215 & 16 & 205 & 3439 \\
\hline Mean & & 17.0 & 2714 & 60.5 & 107.3 & 5.42 & 55.9 & 191.5 & 209 & 15 & 168 & 3242 \\
\hline Min & & 14.1 & 1781 & 57.5 & 98.9 & 3.9 & 53.7 & 172.3 & 195.0 & 15.0 & 115 & 2945 \\
\hline Max & & 20.0 & 3790 & 62.1 & 113.0 & 6.3 & 57.9 & 207.7 & 225.0 & 16.0 & 255 & 3651 \\
\hline
\end{tabular}


Market Segment: Medium Sedans (MED)

Table A2: Functional attributes $\mathbf{f}$, demand $D$, price $P$, soft attributes $\mathbf{S}$

\begin{tabular}{|c|c|c|c|c|c|c|c|c|c|c|c|c|c|}
\hline \multirow[t]{3}{*}{ Brand } & Model & $\mathbf{f}(\mathbf{1})$ & $\mathbf{f}(2)$ & $\mathbf{f}(\mathbf{3})$ & $\mathbf{f}(\mathbf{4})$ & $\mathbf{f}(\mathbf{5})$ & D & $\mathbf{P}$ & $\mathbf{s}(\mathbf{1})$ & $\mathbf{s}(2)$ & $\mathbf{s}(\mathbf{3})$ & $\mathbf{s}(\mathbf{4})$ & $s(5$ \\
\hline & & PV & CV & TC & $\mathbf{F E}^{*}$ & AC & SV & PR & MQ & $\mathrm{CO}$ & ST & MD & SF \\
\hline & & [cft] & {$[\mathrm{cft}]$} & {$[\mathrm{lb}]$} & [mpg] & {$[\mathrm{sec}]$} & {$[\#]$} & {$[\$]$} & $1-5$ & $1-5$ & $1-5$ & $1-5$ & 18 \\
\hline Honda & Accord & 92.7 & 13.6 & 1000 & 28 & 9.42 & 414718 & $\$ 18,890$ & 4 & 4 & 2 & 5 & 4 \\
\hline Toyota & Camry & 101.7 & 16.7 & 2000 & 27 & 9.31 & 390449 & $\$ 18,970$ & 3 & 4 & 2 & 3 & \\
\hline Ford & Taurus & 104.7 & 17 & 1250 & 23 & 10.02 & 353560 & $\$ 19,035$ & 3 & 3 & 3 & 3 & \\
\hline Chevrolet & Impala & 104.5 & 18.6 & 1000 & 25 & 8.76 & 208395 & $\$ 20,325$ & 4 & 4 & 2 & 3 & \\
\hline Pontiac & Grd AM & 92.2 & 14.6 & 1225 & 28 & 10.37 & 182046 & $\$ 17,135$ & 3 & 4 & 2 & 3 & \\
\hline Chevrolet & Malibu & 98.6 & 17.1 & 1000 & 23 & 8.36 & 176583 & $\$ 17,760$ & 3 & 3 & 2 & 4 & \\
\hline Nissan & Altima & 103.2 & 15.6 & 1000 & 26 & 8.11 & 148345 & $\$ 16,649$ & 2 & 3 & 2 & 5 & \\
\hline VW & Jetta & 86.9 & 13 & 1225 & 27 & 11.92 & 145221 & $\$ 16,850$ & 3 & 5 & 3 & 2 & \\
\hline Buick & Century & 101 & 16.7 & 1000 & 23 & 8.96 & 142157 & $\$ 20,535$ & 4 & 3 & 2 & 4 & \\
\hline Pontiac & G Prix & 98 & 16 & 1000 & 23 & 9.00 & 128935 & $\$ 21,230$ & 3 & 3 & 2 & 3 & \\
\hline Chrysler & Sebring & 86.3 & 16.3 & 1000 & 24 & 10.16 & 118459 & $\$ 20,390$ & 3 & 4 & 2 & 2 & \\
\hline Dodge & Stratus & 86.3 & 16.3 & 1000 & 25 & 9.86 & 111125 & $\$ 18,690$ & 3 & 4 & 2 & 2 & \\
\hline Oldsmobile & Alero & 91.2 & 14.6 & 1225 & 27 & 10.01 & 109302 & $\$ 17,805$ & 3 & 3 & 2 & 2 & \\
\hline Dodge & Interpid & 104.5 & 18.4 & 1500 & 23 & 8.07 & 109098 & $\$ 20,810$ & 4 & 3 & 2 & 4 & \\
\hline Mercury & Sable & 102.5 & 16 & 1250 & 23 & 10.02 & 102646 & $\$ 20,020$ & 3 & 3 & 2 & 3 & \\
\hline Nissan & Maxima & 102.5 & 15.1 & 1000 & 22 & 5.87 & 102535 & $\$ 24,699$ & 3 & 5 & 2 & 3 & \\
\hline Saturn & L-100 & 96.9 & 17.5 & 1000 & 27 & 10.31 & 98227 & $\$ 16,370$ & 4 & 4 & 2 & 2 & \\
\hline Subaru & Legacy & 91.4 & 12.4 & 2000 & 23 & 9.37 & 95291 & $\$ 19,295$ & 3 & 3 & 2 & 2 & \\
\hline VW & Passat & 95.4 & 15 & 1225 & 25 & 9.10 & 95028 & $\$ 21,750$ & 4 & 5 & 2 & 2 & \\
\hline Mitsubishi & Galant & 97.6 & 14.6 & 1225 & 24 & 10.08 & 93878 & $\$ 17,707$ & 3 & 3 & 2 & 2 & \\
\hline Toyota & Avalon & 105.6 & 15.9 & 2000 & 24 & 7.57 & 83005 & $\$ 25,845$ & 5 & 5 & 2 & 5 & \\
\hline Chevrolet & M Carlo & 98.2 & 15.8 & 1000 & 25 & 8.64 & 72596 & $\$ 20,425$ & 3 & 4 & 3 & 2 & \\
\hline Hyundai & Sonata & 100 & 14.1 & 1225 & 25 & 10.05 & 62385 & $\$ 15,499$ & 2 & 3 & 2 & 2 & \\
\hline Mazda & 626 & 97.1 & 14.2 & 1225 & 29 & 11.03 & 50997 & $\$ 18,785$ & 3 & 4 & 2 & 3 & \\
\hline Buick & Regal & 101.8 & 16.7 & 1000 & 23 & 8.00 & 49992 & $\$ 23,485$ & 5 & 3 & 2 & 4 & \\
\hline Oldsmobile & Intrigue & 101 & 16.4 & 1000 & 24 & 7.43 & 39395 & $\$ 23,160$ & 4 & 3 & 2 & 3 & \\
\hline Chrysler & Concorde & 107.6 & 18.7 & 1500 & 23 & 8.10 & 32331 & $\$ 22,790$ & 4 & 3 & 3 & 5 & \\
\hline $\mathrm{Kia}$ & Optima & 100 & 13.6 & 1225 & 24 & 9.96 & 25910 & $\$ 14,899$ & 2 & 3 & 2 & 2 & \\
\hline Daewoo & Leganza & 101 & 14.1 & 1225 & 23 & 11.22 & 18347 & $\$ 14,599$ & 3 & 3 & 2 & 2 & \\
\hline Hyundai & XG350 & 102 & 14.5 & 1225 & 21 & 8.76 & 17884 & $\$ 23,999$ & 2 & 5 & 3 & 2 & \\
\hline Mitsubishi & Diamante & 100.9 & 14.2 & 1225 & 20 & 7.81 & 17227 & $\$ 25,687$ & 3 & 3 & 2 & 2 & \\
\hline Mean/Tot. & & 98.5 & 15.6 & 1225.0 & 24.4 & 9.2 & 3796067 & $\$ 19,809$ & 3.3 & 3.6 & 2.1 & 2.9 & \\
\hline Min & & 86.3 & 12.4 & 1000.0 & 20.0 & 5.9 & 17227 & $\$ 14,599$ & 2.0 & 3.0 & 2.0 & 2.0 & \\
\hline $\operatorname{Max}$ & & 107.6 & 18.7 & 2000.0 & 29.0 & 11.9 & 414718 & $\$ 25,845$ & 5.0 & 5.0 & 3.0 & 5.0 & \\
\hline
\end{tabular}




\section{APPENDIX B}

Scatter plots for vehicles in automotive product database (Appendix A)
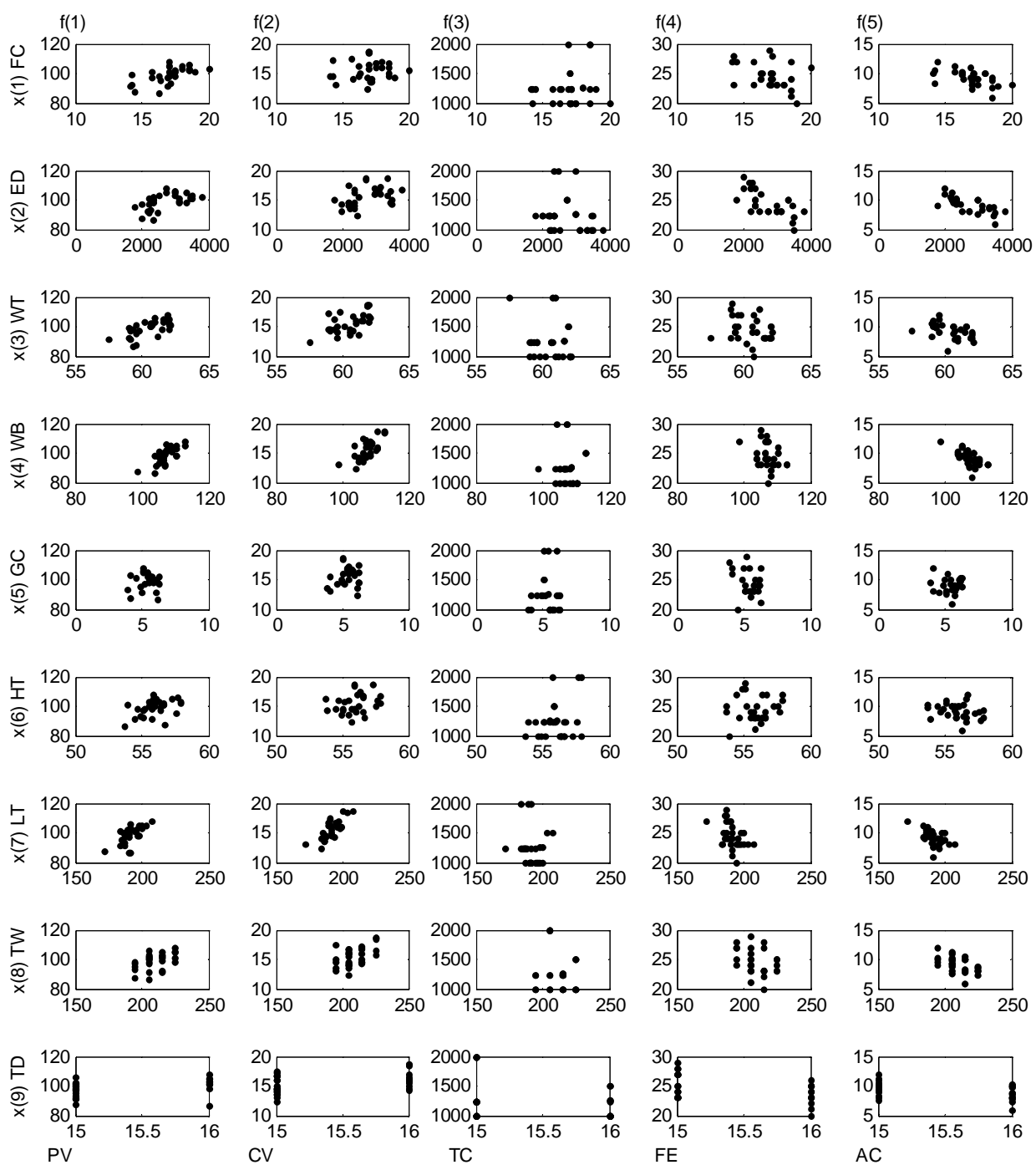

Figure B1: Scatter plots of $\mathbf{x}$ versus $\mathbf{f}$ for medium sedan (MED) market segment 


\section{APPENDIX C}

Design variable bounds given by vehicle product database

Table C1: Overview of design and dependent variable for 7 market segments

\begin{tabular}{|c|c|c|c|c|c|c|c|c|c|c|c|}
\hline Segment & $\begin{array}{r}\mathbf{x}(\mathbf{1}) \\
\mathbf{F C} \\
{[\mathrm{g}]} \\
\end{array}$ & $\begin{array}{c}\mathbf{x}(2) \\
\mathbf{E D} \\
{[\mathrm{ccm}]} \\
\end{array}$ & $\begin{array}{l}\mathbf{x}(3) \\
\mathbf{W T} \\
{[\mathrm{in}]} \\
\end{array}$ & $\begin{array}{l}\mathbf{x}(4) \\
\mathbf{W B} \\
{[\mathrm{in}]} \\
\end{array}$ & $\begin{array}{l}\mathbf{x}(\mathbf{5}) \\
\mathbf{G C} \\
{[\mathrm{in}]} \\
\end{array}$ & $\begin{array}{c}\mathbf{x}(6) \\
\mathbf{H T} \\
{[\mathrm{in}]} \\
\end{array}$ & $\begin{array}{l}\mathbf{x}(7) \\
\mathbf{L T} \\
{[\mathrm{in}]} \\
\end{array}$ & $\begin{array}{c}\mathbf{x}(\mathbf{8}) \\
\mathbf{T W} \\
{[\mathrm{mm}]} \\
\end{array}$ & $\begin{array}{c}\mathbf{x}(\mathbf{9}) \\
\mathbf{T D} \\
{[\mathrm{in}]} \\
\end{array}$ & $\begin{array}{l}\mathbf{y}(\mathbf{1}) \\
\mathbf{H P} \\
{[\mathrm{hp}]} \\
\end{array}$ & $\begin{array}{l}\mathbf{y}(2) \\
\mathbf{C W} \\
{[\mathrm{lbs}]}\end{array}$ \\
\hline SML - avg & 13.37 & 1860 & 57.73 & 99.9 & 5.27 & 55.50 & 172.18 & 187.08 & 14.38 & 122 & 2535 \\
\hline $\mathrm{SML}-\min$ & 11.9 & 1468 & 55.30 & 93.3 & 3.90 & 53.00 & 161.10 & 175.00 & 13.00 & 92 & 2035 \\
\hline SML - max & 15.9 & 2457 & 59.60 & 105.0 & 7.70 & 59.40 & 182.00 & 205.00 & 16.00 & 180 & 2965 \\
\hline MED - avg & 17.0 & 2714 & 60.5 & 107.3 & 5.42 & 55.9 & 191.5 & 209 & 15 & 168 & 3242 \\
\hline MED - min & 14.1 & 1781 & 57.5 & 98.9 & 3.9 & 53.7 & 172.3 & 195.0 & 15.0 & 115 & 2945 \\
\hline MED - max & 20.0 & 3790 & 62.1 & 113.0 & 6.3 & 57.9 & 207.7 & 225.0 & 16.0 & 255 & 3651 \\
\hline LRG - avg & 19.40 & 3737 & 61.57 & 112.1 & 5.40 & 56.57 & 197.99 & 221.00 & 16.13 & 243 & $\mathbf{3 7 5 4}$ \\
\hline $\mathrm{LRG}-\min$ & 16.9 & 1985 & 57.2 & 102.6 & 3.9 & 53.6 & 182.3 & 205.00 & 15.00 & 185 & 3220 \\
\hline LRG - max & 23.7 & 4605 & 63.4 & 121.5 & 6.7 & 58.9 & 215.3 & 245.00 & 18.00 & 340 & 4376 \\
\hline SPT - avg & 15.05 & 2743 & 58.54 & 98.2 & 4.68 & 51.36 & 170.25 & 211 & 15.83 & 200 & 2879 \\
\hline $\mathrm{SPT}-\min$ & 12.70 & 1781 & 55.60 & 89.2 & 4.00 & 47.70 & 153.00 & 185 & 14.00 & 115 & 2195 \\
\hline SPT - max & 18.50 & 5665 & 61.90 & 106.4 & 6.00 & 56.00 & 193.50 & 245 & 17.00 & 350 & 3323 \\
\hline VAN - avg & 21.70 & 3397 & 63.17 & 114.8 & 6.17 & 68.89 & 194.24 & 214.33 & 15.13 & 189 & 4041 \\
\hline $\mathrm{VAN}-\min$ & 19.80 & 2429 & 60.60 & 111.2 & 4.30 & 64.20 & 186.90 & 205.00 & 15.00 & 150 & 3699 \\
\hline VAN - max & 27.00 & 4300 & 66.10 & 120.7 & 8.70 & 75.00 & 201.50 & 225.00 & 16.00 & 240 & 4709 \\
\hline SUV - avg & 21.06 & 3491 & 61.61 & 108.7 & 8.14 & 70.68 & 185.39 & 237.16 & 15.95 & 202 & 4075 \\
\hline $\mathrm{SUV}-\min$ & 14.70 & 1983 & 55.00 & 92.9 & 6.30 & 65.00 & 155.40 & 195.00 & 15.00 & 120 & 2777 \\
\hline SUV - max & 44.00 & 5408 & 68.40 & 137.1 & 10.20 & 77.20 & 226.70 & 275.00 & 17.00 & 300 & 6650 \\
\hline TRK - avg & 22.8 & 3457 & 61.7 & 119.1 & 8.2 & 68.2 & 203.3 & 235.0 & 15.6 & 180 & 3774 \\
\hline $\mathrm{TRK}-\min$ & 15.8 & 2189 & 54.4 & 103.3 & 6.7 & 62.0 & 184.4 & 205.0 & 15.0 & 120 & 2750 \\
\hline TRK - max & 31.0 & 5326 & 68.0 & 131.1 & 10.4 & 74.4 & 221.7 & 265.0 & 17.0 & 285 & 5437 \\
\hline
\end{tabular}

